\title{
基于靶标结构及作用机制的抗艾滋病药物研究进展
}

\author{
陈 超 $a, b$ \\ 胡晓东 $b$ \\ 王春喜 $b$ \\ 蓝文贤 $b$ 吴小余*, $a$ 曹春阳 $*, b$ \\ ( ${ }^{a}$ 上海大学理学院化学系 上海 200444) \\ ( $b$ 中国科学院上海有机化学研究所 生命有机化学国家重点实验室 上海 200032)
}

\begin{abstract}
摘要 人体免疫缺陷病毒(Human immunodeficiency virus, HIV)是一种主要以 $\mathrm{CD} 4^{+} \mathrm{T}$ 淋巴细胞和巨喍细胞等免疫细胞 为靶点的感染性逆转录病毒, HIV 感染的最终阶段为出现获得性免疫缺陷综合征(Acquired immunodeficiency syndrome, AIDS). HIV 迄今为止已经夺去近 3300 万人生命, 是全球最大的公共卫生挑战之一. 自从抗逆转录病毒治疗 (Antiretrovial therapy, ART)出现以后, 抗逆转录药物的联合使用使艾滋病从致死性疾病变成慢性可控性疾病. 为了开 发新的抗艾滋病药物, 基于病毒复制周期中的不同靶标将近年发展的抗艾滋病药物进行分类简述, 重点关注于药物的 作用机制研究、临床应用现状及未来发展方向.
\end{abstract}

关键词 抗逆转录病毒药物; 人体免疫缺陷病毒; 获得性免疫缺陷综合征; 长效抑制剂

\section{Structure- and Mechanism-Based Research Progress of Anti- acquired Immune Deficiency Syndrome Drugs}

\author{
Chen, Chao ${ }^{a, b} \quad \mathrm{Hu}$, Xiaodong $^{b} \quad$ Wang, Chunxi $^{b} \quad$ Lan, Wenxian $^{b}$ \\ Wu, Xiaoyu*,a Cao, Chunyang*,b \\ ( ${ }^{a}$ College of Science, Shanghai University, Shanghai 200444) \\ ( ${ }^{b}$ State Key Lab of Bio-organic and Natural Products Chemistry, Shanghai Institute of Organic Chemistry, \\ Chinese Academy of Sciences, Shanghai 200032)
}

\begin{abstract}
Human immunodeficiency virus (HIV) is a retrovirus that mainly targets immune cells such as $\mathrm{CD} 4{ }^{+} \mathrm{T}$ lymphocytes and macrophages, which eventually leads to acquired immunodeficiency syndrome (AIDS). HIV continues to be a major global public health challenege, having killed almost 33 million lives so far. Since the emergence of antiretroviral therapy (ART), the combination of antiretroviral drugs has transformed HIV-1 infection, once a fatal illness, into a manageable chronic condition. Based on different targets during the virus replication cycle, anti-AIDS drugs in recent years were classified with focus on the mechanism elucidation, current clinical application and future direction.

Keywords antiretroviral drug; human immunodeficiency virus (HIV); acquired immune deficiency syndrome (AIDS); long-acting inhibitor
\end{abstract}

人体免疫缺陷病毒(Human immunodeficiency virus, $\mathrm{HIV})$ 是一种主要以 $\mathrm{CD} 4^{+} \mathrm{T}$ 淋巴细胞和巨噬细胞等免疫 细胞为靶点的感染性逆转录病毒, HIV 感染的最终阶段 为出现获得性免疫缺陷综合征(Acquired immunodeficiency syndrome, AIDS). 自 1981 年美国首例艾滋病患
者被报道至 2019 年年底, 全球约有 3800 万 HIV 携带者, HIV 至今仍是全球最大的公共卫生挑战之一[1]. 1984 年 Klatzmann 等[2]发现 HIV 进入人体后能选择性地进攻具 有 $\mathrm{CD} 4$ 受体的淋巴细胞, 以 $\mathrm{CD} 4^{+} \mathrm{T}$ 淋巴细胞为主, 进 而阻断细胞免疫和体液免疫过程造成免疫缺陷，最终导

\footnotetext{
* Corresponding authors. E-mail: wuxy@shu.edu.cn; ccao@mail.sioc.ac.cn

Received December 22, 2020; revised March 12, 2021; published online April 29, 2021.

Project supported by the National Key Research and Development Program of Ministry of Science and Technology (Nos. 2017YFE0108200, 2016YFA0502302), the Chinese Academy of Sciences Strategic Priority Research Program (No. XDB20000000) and the National Natural Science Foundation of China (Nos. 21778065, 21807105, 21977110, 91753119).

科技部重点研发(Nos. 2017YFE0108200, 2016YFA0502302)、中国科学院战略性先导科技专项(No. XDB20000000)及国家自然科学基金(Nos. 21778065, $21807105,21977110,91753119)$ 资助项目.
} 
致机会性感染和癌症.

HIV 为单链 RNA 病毒, 分为两种亚型: HIV-1 和 HIV-2. 目前大约 $95 \%$ 的 HIV 感染者是属于 HIV-1 的感 染. HIV-1 是从欧洲和美洲感染者中分离出的毒株, 其 致病力强, 死亡率高; 而 HIV-2 的感染局限于在西非地 区，相比 HIV-1 而言, HIV-2 具有更低的病毒载量, 且死 亡率较低 ${ }^{[3]}$. 现大部分 HIV 病毒研究主要是针对 HIV-1 进行.

HIV 属于灵长类慢病毒家族的逆转录病毒, 其基因 组是两条相同的正链 RNA, 每条 RNA 长约 9.2 9.8 $\mathrm{kb}$ (图 1). 病毒基因组主要含有三种结构基因: $g a g$ 基因、 $p o l$ 基因和 $e n v$ 基因; 两种调节基因: tat 基因和 $r e v$ 基因; 五种辅助基因: nef 基因、 vif 基因、 $v p r$ 基因、 $v p u$ 基因 和 $v p x$ 基因 ${ }^{[4]}$. 其中 $g a g$ 基因编码病毒的核心蛋白; pol 基因编码病毒复制所需要的酶(逆转录酶、整合酶和蛋 白酶); $e n v$ 基因编码的病毒包膜蛋白是 HIV 免疫学诊断 的主要检测抗原; $v p u$ 基因只存在于 HIV-1, 而 $v p x$ 基因 仅在 HIV-2 中 ${ }^{[5]}$.
$\mathrm{HIV}$ 病毒粒子是直径 $100 \mathrm{~nm}$ 的二十面体立体对称

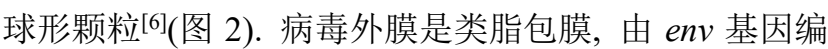
码的外膜蛋白 gp120 和跨膜蛋白 gp41 通过非共价键结 合为异源二聚体，三个异源二聚体组装成三聚体刺突铆 定在病毒包膜上. 基质蛋白(p17)形成的球状基质在病 毒包膜内层起稳定作用. 核心抗原(p24)形成的衣壳内 含有两条相同的 RNA 正链、逆转录酶、整合酶、蛋白 酶以及其他来自宿主的细胞成分 ${ }^{[7]}$.

三十多年来, 全球科学家对 HIV 病毒自身特点以及 其感染途径进行了认真系统的深入研究，找到了控制或 预防艾滋病的有效方法. 抗逆转录病毒治疗 (Antiretrovial therapy, ART)方法出现后，抗逆转录药物的联合 使用(即鸡尾酒疗法)极大地改善了患者的生活水平，使 艾滋病从致死性疾病变成慢性可控性疾病，不再谈“艾” 色变了 ${ }^{[8]}$. 但 ART 并不能完全消除人体内的艾滋病病毒 且治疗价格昂贵，长期服用可能引起严重的副作用。由 于 HIV 具有高复制和高变异等特点, 若单一药物治疗可 造成耐药性，所以临床上一般将 $24 \sim 27$ 种经批准的

a

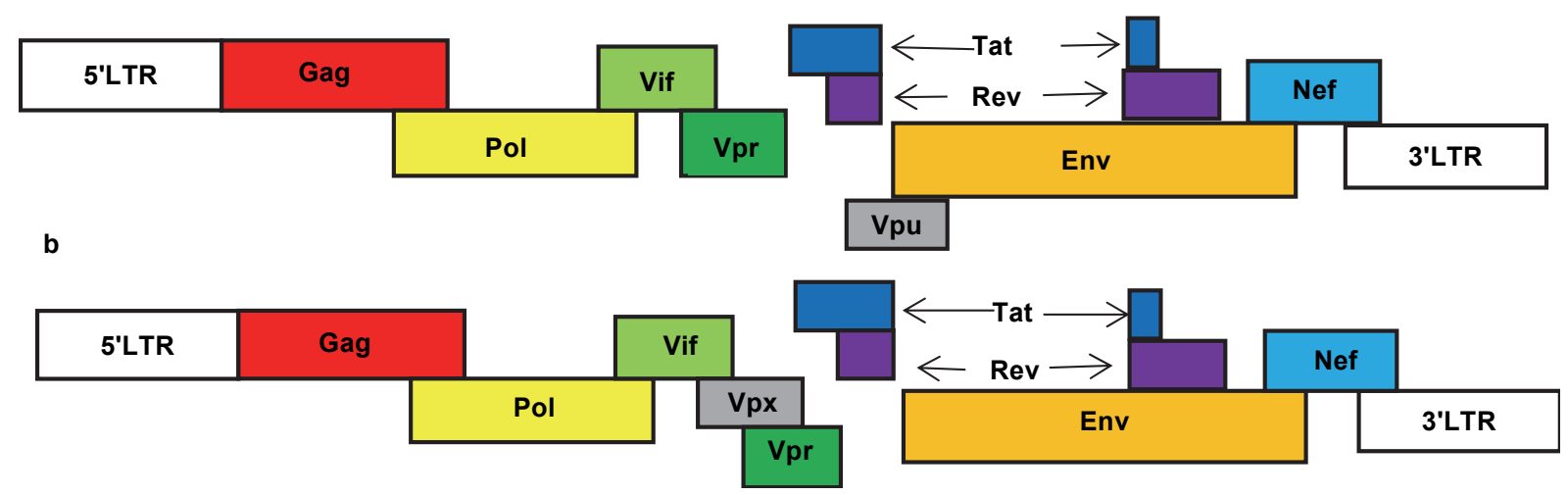

图 1 (a) HIV-1 和(b) HIV-2 病毒基因组结构

Figure 1 Organization of the (a) HIV-1 and (b) HIV-2 genomes

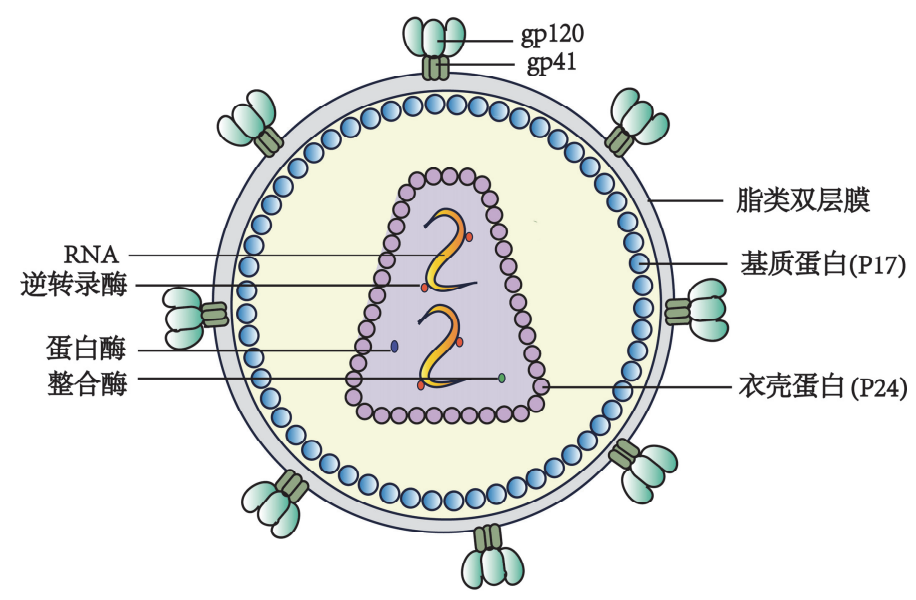

图 $2 \mathrm{HIV}-1$ 病毒粒子结构示意图

Figure 2 Structure of the HIV-1 particle 
药物联合使用以达到高效抗逆转录病毒治疗 (Highly active antiretroviral therapy, HAART $)^{[9]}$. 开发 HIV 疫苗是 预防艾滋病传播的有效方法, 但至今尚未研究出安全有 效的疫苗, 所以抗逆转录病毒药物仍是当前艾滋病治疗 的重要手段. 本文综述近些年抗艾滋病药物及其作用机 制的相关研究进展, 希望能够对相关研究人员研究新型 艾滋病药物有所帮助.

\section{1 抗艾滋病病毒药物}

HIV 在人体的复制过程主要包括病毒进攻细胞和 融合、反转录和整合、转录转译以及组合出芽 (图 3) ${ }^{[5,10]}$. (1)病毒进攻细胞和融合: 成熟病毒粒子上的 gp120 与 gp41 三聚体结构介导病毒与宿主细胞结合. gp120 与 CD4 受体结合后发生结构变化, 随后又与趋化因子受体 (CCR5 或者 CXCR4)结合使病毒粒子与靶细胞膜结合更 加紧密, 暴露出的 gp41 发生构型变化形成 6 股 $\alpha$ 螺旋束 核心结构(6-HB), 介导膜融合和病毒进入细胞. (2)反转 录和整合: 病毒 RNA 在逆转录酶的作用下在细胞质中 逆转录形成 RNA/DNA 杂交双链, 核糖核酸酶 $\mathrm{H}$ 分解 RNA 链, 转录酶完成 DNA 互补, 形成完整的双螺旋 DNA 后由整合酶整合到细胞基因组中. (3)转录转译以
及组合出芽: 病毒 mRNA 转移到细胞质中翻译形成新 病毒粒子的结构蛋白. $g a g$ 基因编码的前体蛋白(P55)经 HIV 蛋白酶的裂解形成病毒的核衣壳蛋白(P7)、内膜蛋 白(P17)和衣壳蛋白(P24). 包膜糖蛋白前体 gp160 也在 蛋白酶的作用下裂解为 gp120 和 gp41, 并被转运、集结 于细胞膜. 未成熟的病毒粒子向细胞表面迁移, 随后将 病毒释放到细胞外, 最终形成新的具有感染性的病毒粒 子[5].

基于 HIV 病毒复制周期, 药物化学家采用了多种途 径阻断病毒复制从而抑制 HIV 感染. 1987 年第一个美国 食品药品监督管理局(FDA)批准生产的抗艾滋病药物齐 多夫定(Zidovudine, AZT)面世后, 一系列抗 HIV 药物研 究逐渐发展起来. 按照抗 HIV 药物在不同靶点上的不同 作用机制, 主要分为核苷类反转录酶抑制剂(Nucleoside reverse transcriptase inhibitors, NRTIs)、非核苷类反转录 酶抑制剂(Non-nucleoside reverse transcriptase inhibitors, NNRTIs)、蛋白酶抑制剂(Protease inhibitors, PIs)、整合 酶抑制剂(Integrase inhibitors, INSTIs)、侵入抑制剂 (Entry inhibitors)以及其他新型抑制剂(表 1). 为了更好 了解它们, 下面对它们进行一一介绍.

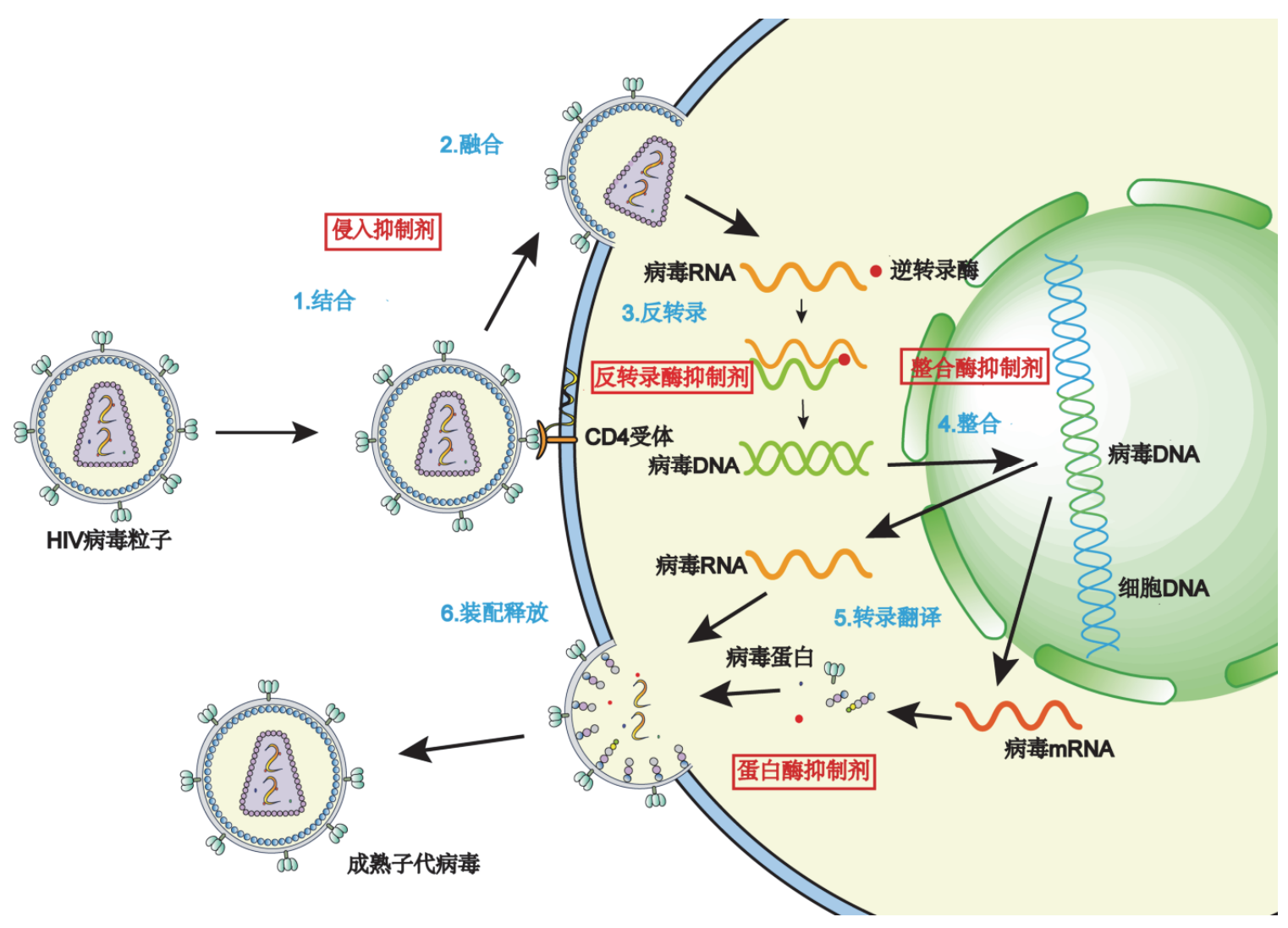

图 $3 \mathrm{HIV}$ 体内复制过程

Figure 3 HIV replication cycle in vivo 
表 1 近年已上市和处于临床研究阶段的抗艾滋病药物

Table 1 HIV antiretroviral drugs listed and in clinical development recently

\begin{tabular}{|c|c|c|c|c|}
\hline & 药物类型 & 药物名称 & 研发状况 & 公司 \\
\hline & \multirow{2}{*}{ 核苷类反转录酶抑制剂 } & EFdA (MK-8591, Islatravir ) & III期临床 & Merck \& Co \\
\hline & & GS-9131 (Rovafovir Etalafenamide) & 临床前期 & Gilead Sciences \\
\hline & \multirow{2}{*}{ 非核苷类反转录酶抑制剂 } & Doravirine (MK-1439) & 已上市 & Merck \& Co \\
\hline & & Elsulfavirine (VM1500) & II/III期临床 & Viriom \\
\hline & 蛋白酶抑制剂 & Darunavir & 已上市 & Tibotec \\
\hline & 整合酶抑制剂 & Cabotegravir (GSK1265744) & 已上市 & ViiV Healthcare \\
\hline \multirow{6}{*}{ 侵入抑制剂 } & 附着抑制剂 & Fostemsavir (GSK-3684394, BMS-663068) & 已上市 & ViiV Healthcare \\
\hline & 附着后抑制剂 & Trogarzo (ibalizumab-uiyk) & 已上市 & TaiMed Biologics \\
\hline & 趋化因子受体抑制剂 & Thioraviroc & I期临床 & $\begin{array}{l}\text { 上海药物研究所 } \\
\text { 昆明动物研究所 }\end{array}$ \\
\hline & 融合抑制剂 & Albuvirtide & 已上市 & 前沿生物 \\
\hline & 衣壳抑制剂 & Lenacapavir (GS-6207) & II/III期临床 & Gilead Sciences \\
\hline & 成熟抑制剂 & GSK2838232 & II期临床 & GlaxoSmithKline \\
\hline
\end{tabular}

\section{1 核苷类反转录酶抑制剂}

核苷类反转录酶抑制剂(NRTIs)进入到感染细胞中 被磷酸化, 其活性三磷酸核苷衍生物结合于病毒 DNA 链 3'末端, 竞争性抑制病毒逆转录酶活性, 通过阻碍磷 酸二酯键的形成从而终结 DNA 链合成 ${ }^{[11]}$. 如图 4 所示, FDA 批准的 NRTIs 药物有齐多夫定(Zidovudine, AZT)、 阿巴卡韦(Abacavir, ABC)、去羟肌苷(Didanosine, DDI)、 拉米夫定(Lamivudine, 3TC)、司他夫定(Stavudine, D4T)、 恩区他滨(Emtricitabin, FTC)、富马酸替诺福韦二吡呋酯 (Tenofovir disproxil fumarate, TDF)和富马酸丙酚替诺福 韦(Tenofovir alafenamide fumarate, TAF). Tenofovir disproxil fumarate 与 Tenofovir alafenamide fumarate 均为替 诺福韦(Tenofovir, TFV)前药. 与 Tenofovir disproxil fumarate 相比, Tenofovir alafenamide fumarate 只需要少于 十分之一的剂量便可获得相似的抗病毒效果, 具有更好 的血浆稳定性且肾、骨毒性更小, 所以在临床上 Tenofovir alafenamide fumarate 逐渐取代 Tenofovir disproxil fumarate 成为新兴的 NRTIs ${ }^{[12]}$. 目前可用的 NRTIs 均存在很大的副作用, 于是对核苷类化合物结构改造成 了研究的主要方向.

\subsubsection{EFdA (MK-8591, Islatravir)}

默克公司研制的新一代 NRTIs 药物 EFdA (MK8591, Islatravir)在野生型 HIV 和耐药 HIV 突变体中展现 出极好的抗病毒活性和低毒性(图 5a) ${ }^{[13]}$. 与其他 NRTIs 相比, 具有 $3^{\prime}$ 位羟基的 $\mathrm{EFdA}$ 在结构上与逆转录酶的天 然底物更加相似, 从而与逆转录酶结合能力更强. 由于 腺嘌呤环上 2 位氟的作用, 改变了环上电子分布, 从而 抑制腺苷脱氨酶水解 ${ }^{[14-15]}$, 而且 4 '位的乙炔基也有助于 降低 EFdA 降解 ${ }^{[16]}$. 这些结构优势使 EFdA 更加稳定, 是其成为长效抗病毒药物的关键因素. 磷酸化活性代谢 物 EFdA-TP (EFdA-triphosphate) 对逆转录酶结合位点有<smiles>[Z20][C@@H]1C[C@H](CO)O[C@H]1n1cnc2c(=O)[nH]cnc21</smiles>

Didanosine<smiles>Cc1cn([C@H]2C=C[C@@H](CO)O2)c(=O)[nH]c1=O</smiles><smiles>Nc1nc(NC2CC2)c2ncn(C3C=C[C@@H](CO)C3)c2n1</smiles><smiles>Nc1ccn(C2CSC(CO)O2)c(=O)n1</smiles>

Lamivudine

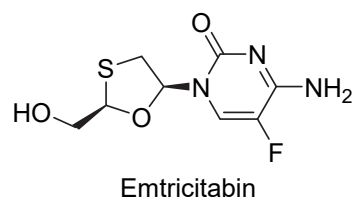

Emtricitabin<smiles>CC(C)OC(=O)OCOP(=O)(COC(=O)OC(C)C)OCOC(=O)OC(C)Cn1cnc2c(N)ncnc21</smiles>

图 4 FDA 批准的 NRTIs 药物

Figure 4 Structures of FDA-approved NRTIs

高度亲和力, 且两者结合力大于逆转录酶与天然 $d N T P$ 底物结合力 ${ }^{[17]}$. 早期的分子模型 ${ }^{[18]}$ 以及近年晶体结 构 ${ }^{[19]}$ 表明 EFdA-TP 的 4'位的乙炔基结合于酶的疏水口 袋中, 有助于 EFdA-TP 与逆转录酶的高度结合(图 5b). 
<smiles>C=C[C@@H](O)[C@H]1CC(n2cnc3c(N)nc(F)nc32)O[C@@]1(CO)C(=O)O</smiles><smiles>C=C[C@@]1(COP(=O)(O)OP(=O)(O)OP(=O)(O)O)OC(n2cnc3c(N)nc(F)nc32)CC1O</smiles>

b

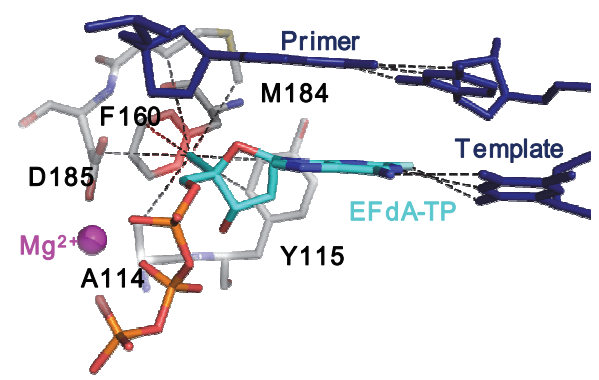

图 5 (a) EFdA 化学结构和(b) EFdA-TP 与逆转录酶-DNA 复 合物晶体结构 ${ }^{[19]}$ (PDB 号 $\left.5 \mathrm{~J} 2 \mathrm{M}\right)$

Figure 5 (a) Chemical structure of EFdA and (b) crystal structure of EFdA-TP binding to reverse transcriptase-DNA complex $^{[19]}$ (PDB code 5J2M)

DNA is in dark blue, $\mathrm{Mg}^{2+}$ ion is indicated by magenta sphere, and hydrogen bonds are depicted by black dashed lines
EFdA 的关键结构特征为具有炔基取代的 4'-C 立体 中心, 其次 $3^{\prime}$ 位的着基和 $N$-糖基化的立体选择使 $\mathrm{EFdA}$ 成为功能强大且独特有趣的药物合成目标. 2017 年美国 默克公司提出一种新颖的 $\mathrm{EFdA}$ 对映选择性合成路线

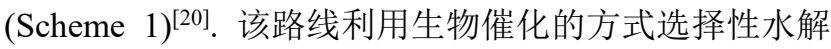
单个酯基实现二乙酸酯 2 的去对称化，在优化条件下以 95\%的收率和 $96 \%$ 对映体过剩率生成 4'-C 立体中心. 在 之后的反应中酮酯化合物 6 通过不对称转移氢化反应完 成 3'位羟基的立体选择性，以 $95 \%$ 收率和极高的非对映 选择性 $(d r>99: 1)$ 获得所需的 $\beta$-差弪基酯 7 .

糖基化过程则面临着端基异构体选择性合成与分 离高纯度目的产物的巨大挑战：通过优化条件下的 Vorbruggen 糖基化反应实现了较高的端基异构体选择 性; 并在省去水淬灭处理步骤后直接分离得到高纯度的 $\beta$-端基异构体 12. 最终脱保护获得 EFdA. 该合成路线 从廉价的 1,3-二乙酰氧基丙酮出发，适用于制备 $10 \mathrm{~kg}$ 以上的 $\mathrm{EFdA}$, 路线总收率为 $17 \%$. 这种利用酶生物催 化的不对称合成方法经济且可靠, 但步骤相对较长且糖 基化反应效果不太理想.

2020 年 Nawrat 等 ${ }^{[21]}$ 研发了一种高效且高立体选择 性的九步合成路线(Scheme 2). 从 2-脱氧- $D$ 核糖 3 步衍 生的可烯醇化酮 13 出发, 在优化条件下利用乙炔类亲 核试剂进行反应得到化合物 14, 其收率 $72 \%$ 且非对映



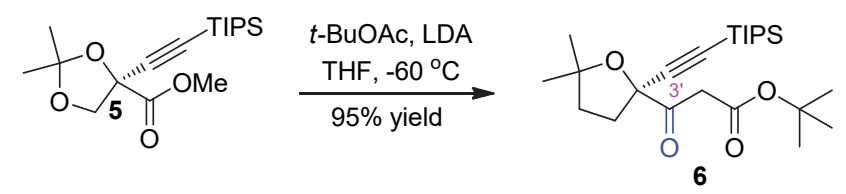

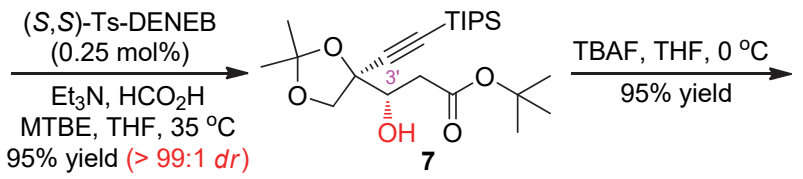<smiles>C#C[C@]1([C@@H](O)CC(=O)OC(C)(C)C)COC(C)(C)O1</smiles>

(1) $\mathrm{HCl}$ (concd.)

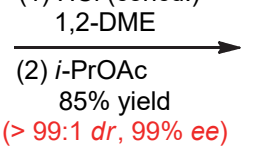<smiles>C#C[C@]1(CO)OC(=O)C[C@H]1O</smiles>

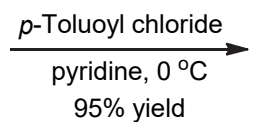

$95 \%$ yield
(1) Red-Al, toluene

DCM, $-60{ }^{\circ} \mathrm{C}$

(2) $\mathrm{Ac}_{2} \mathrm{O}, \mathrm{Et}_{3} \mathrm{~N}, \mathrm{DMAP}$ $80 \%$ yield

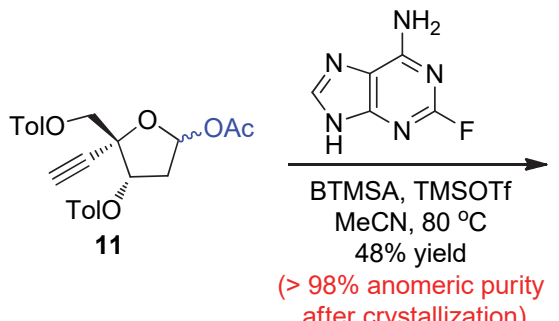<smiles>C#C[C@]1(CO)O[C@@H](n2cnc3c(NS(C)(=O)=O)nc(F)nc32)C[C@H]1O</smiles><smiles>C#C[C@]1(CO)O[C@@H](n2cnc3c(N)nc(F)nc32)C[C@H]1O</smiles>

图式 $1 \mathrm{EFdA}$ 的酶催化不对称合成

Scheme 1 Enantioselective synthesis of EFdA via enzymatic desymmetrization 

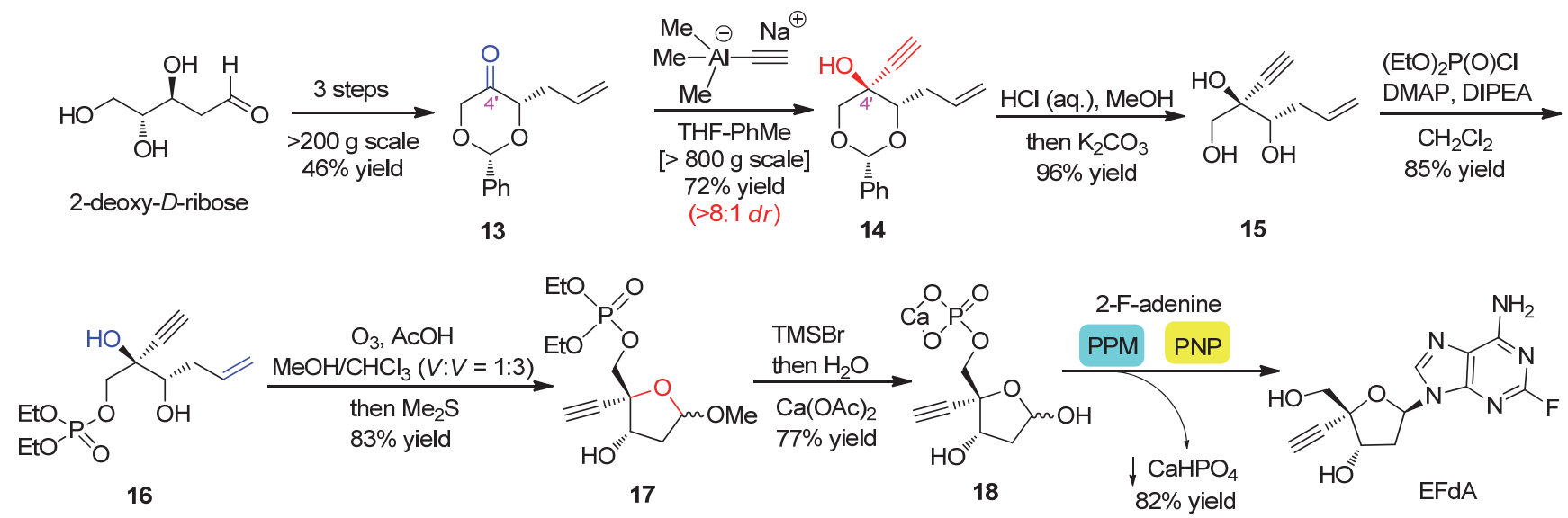

图式 2 从脱氧核糖九步立体选择性合成 $\mathrm{EFdA}$

Scheme 2 Nine-step stereoselective synthesis of EFdA from deoxyribose

异构率为 $8.4: 1$. 随后完成苯亚甲基缩醛的脱保护以及 伯醇的区域选择性磷酸化, 并且对末端烯烃进行选择性 臭氧分解生成甲基糖苷混合物 17. 使用溴代三甲基硅 烷脱烷基后用水淬灭，但因产物中过多的澳离子会影响 后续酶促糖基化效率, 故利用 $\mathrm{Ca}(\mathrm{OAc})_{2}$ 生成高稳定性 的不溶钙盐 18 分离出来. 最终利用嘌呤核苷磷酸化酶 (Purine nucleoside phosphorylase, PNP)和磷酸戊糖变位 酶(Phosphopentomutase, PPM)实现酶促糖基化级联反应 从而以高于 $99 \%$ 纯度和 $82 \%$ 产率获得产物. 该合成特色 在于利用乙炔基亲核试剂进行非对映选择性合成 4'-C 立体中心, 对末端烯烃进行化学选择性臭氧分解以及生 物催化糖基化级联反应，明显缩短了合成步骤，从酮 13 开始的后六步总产率为 36\%.

在体外实验中发现, EFdA 可在皮摩尔 $(\mathrm{pmol} / \mathrm{L})$ 浓度 范围内对活化的外周血单核细胞(Peripheral blood mononuclear cell, PBMCs)和 MT4 细胞表现出显著的抗病毒 效果 ${ }^{[22]}$, 其药效是 Tenofovir 的 8400 倍, 比 Emtricitabin 强 1000 倍 ${ }^{[23]} .2017$ 年针对 30 名 HIV-1 感染者的 EFdA 剂量范围的研究表明其活性三磷酸酯在外周血单核细 胞内半衰期为 $78.5 \sim 128 \mathrm{~h}$, 同时低至 $0.5 \mathrm{mg}$ 的单剂量 也具有强大的抗病毒活性 ${ }^{[24]}$. 在恒河猴上进行的实验 研究表明 EFdA 对于反复暴露于 SIV 的粘膜有着高度保 护作用, 这说明 EFdA 具有暴露前预防(Pre-exposure Prophylaxis, PrEP)的潜在用途 ${ }^{25-26]}$. 总而言之, EFdA 能 够有效延长半衰期且具有极高的抗病毒活性, 可有效解 决 HIV-1 患者在 HAART 治疗中依从性差的问题, 使之 调整为每周口服一次. 近日默克公司在艾滋病毒预防研 究会议 (HIVR4P 2021) 上公布了 IIa 期临床试验 (NCT04003103)新的中期数据, 完整数据将于 2021 年底 公布并且部分III期临床试验现已陆续开展.

\subsubsection{GS-9131 (Rovafovir Etalafenamide)}

吉利德公司开发设计的 GS-9131 (Rovafovir Etalafenamide)是一种具有发展潜力的 NRTIs 药物. 基于 d4AP 骨架上 2 '位氟化后的腺苷衍生物 GS-9148, 其抗病 毒药效增强且线粒体毒性降低. GS-9131 是化合物 GS-9148 的口服生物可利用的磷酸盐前体 ${ }^{[27]}$ (图 6a). 在 此, ProTide (Prodrug nucleotide)技术将核苷单磷酸的磷 酸根改造为 $N$-氨基酸酯和酚氧基取代的磷酰胺或膦酰 胺，降低了化合物极性，增加亲脂性从而增加细胞透膜 性，更能大大提高其代谢活化效率使其高效地在细胞内 转化为相应的核苷三磷酸 (Nucleoside triphosphate, NTP) ${ }^{[28-29]}$. 这种前药技术不仅运用于 GS-9131 的合成 ${ }^{[27]}$, 而且在 Tenofovir 的前药 Tenofovir alafenamide fumarate 中也有所体现. 在早期的临床研究中 GS-9148 表现出低 细胞毒性、良好的耐药性以及药代动力学特征 ${ }^{[30]}$. GS-9148 对 HIV 野生型具有抗逆转录病毒活性 $\left(\mathrm{EC}_{50}=\right.$ $12 \mu \mathrm{mol} / \mathrm{L}$ ), 与大多数临床逆转录酶抑制剂不同, 其对 K65R、M184V、L74V 耐药突变体也有着抗病毒活性 ${ }^{[31]}$. 但是非常罕见的 Q151L 突变体可能对 GS-9131 具有耐 药性, GS-9148 的 2 位氟可能与 Q151L 突变体的侧链存 在空间位阻，从而阻碍了抑制剂活性形式 GS-9148-DP (GS- 9148-diphosphate)和逆转录酶结合(图 $6 \mathrm{~b}, 6 \mathrm{c})^{[32]}$. 基 于这些优点，一种可注射的 GS-9131 制剂目前正在临床 开发中, 但是其作为抗 HIV 长效抑制剂的潜力仍需要进 一步临床研究.

\section{2 非核苷类反转录酶抑制剂(NNRTIs)}

NNRTIs 药物一般对 HIV-1 有抗病毒活性，而对 HIV-2 无活性. NNRTIs 结合于逆转录酶催化位点附近并 导致酶构象变化，与底物的结合位点不同，故 NNRTIs 能够非竞争性抑制病毒逆转录酶. NNRTIs 的副作用一 
a<smiles>Nc1ncnc2c1ncn2-c1ccc(OP(O)(O)(O)O)o1</smiles><smiles>Nc1ncnc2c1ncn2C1OC(OP(=O)(O)O)C=C1F</smiles>

GS-9148<smiles>CCOC(=O)C[C@H](C)P(=O)(COC1C=C(F)C(n2cnc3c(N)ncnc32)O1)Oc1ccccc1</smiles>
GS-9131

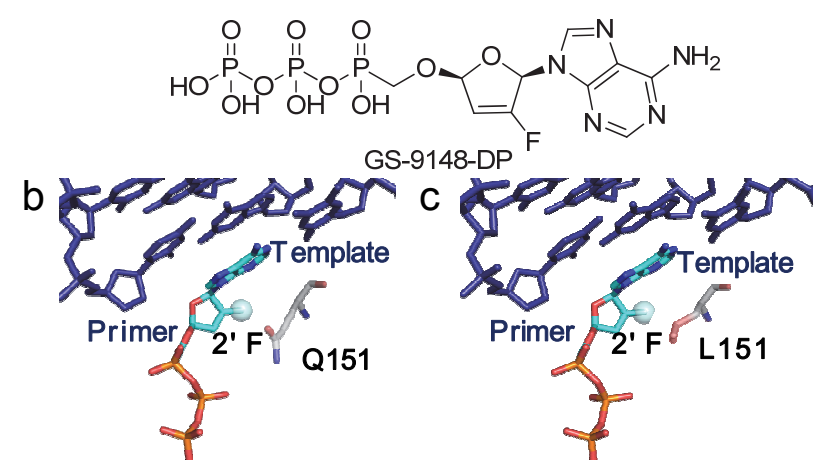

图 6 (a) d4AP, GS-9148, GS-9131 以及 GS-9148-DP 化学结 构、(b) GS-9148-DP 与逆转录酶-DNA 复合物晶体结构(PDB 号 3KK1 ${ }^{[33]}$ 和(c) GS-9148-DP 与逆转录酶 Q151L-DNA 复合物 晶体结构

Figure 6 (a) Chemical structure of d4AP, GS-9148, GS-9131 and GS-9148-DP, (b) crystal structure of GS-9148-DP binding to reverse transcriptase-DNA complex(PDB code $3 \mathrm{KK} 1)^{[33]}$ and (c) crystal structure of GS-9148-DP binding to reverse transcriptase Q151L mutant-DNA complex

Nucleic acids is in dark blue, 2'-fluorine atom is indicated by light blue sphere.

般小于核苷类似物, 其主要缺点是用药后病毒耐药性发 展较快, 故 NNRTIs 通常与其他药物联合治疗 HIV 感 染 ${ }^{[34]}$. 如图 7 所示, FDA 批准的 NNRTIs 药物有: 奈韦拉 平(Nevirapine, NVP)、地拉夫定(Delavirdine, DLV)、依 法韦仑(Efavirenz, EFV)、依曲韦林(Etravirine, ETR)、利 匹韦林(Rilpivirine, RPV)和多拉维林(Doravirine, DOR). 1998 年上市的第一代 NNRTIs 药物 Efavirenz 是一种手 性药物且只有 $S$ 型分子具有抗病毒活性, 因此高对映选 择性合成 Efavirenz 一直以来吸引着有机合成化学家们, 现已出现了一系列的 Efavirenz 合成方法 ${ }^{[35]}$. 近期 Meggers 等 ${ }^{[36]}$ 利用已知的手性钉催化剂高效构建 Efavirenz 关键中间体, 该合成路线产率高且对映选择性 高.<smiles>COS(=O)(=O)c1ccc2[nH]c(C(=O)N3CCN(c4ncccc4NC(C)C)CC3)cc2c1</smiles><smiles>Cc1cc(/C=C/C#N)cc(C)c1Nc1ccnc(Nc2ccc(C#N)cc2)n1</smiles><smiles>CC1(C#CC2CC2)OC(=O)Nc2ccc(Cl)cc21</smiles>

Efavirenz<smiles>Cc1cc(C#N)cc(C)c1Oc1nc(Nc2ccc(C#N)cc2)nc(N)c1Br</smiles>

Etravirine<smiles>Cn1c(Cn2ccc(C(F)(F)F)c(Oc3cc(Cl)cc(C#N)c3)c2=O)n[nH]c1=O</smiles>

Doravirine
图 7 FDA 批准的 NNRTIs 药物

Figure 7 Structures of FDA-approved NNRTIs

\subsubsection{Doravirine (DOR, MK-1439)}

美国默沙东开发设计的 Doravirine (MK-1439, DOR)于 2018 年被 FDA 批准上市. 作为一种变构抑制 剂, Doravirine 结合于逆转录酶的 p66 亚基的疏水口袋, 导致逆转录酶构象变化从而抑制 HIV-1 DNA 合成. Doravirine 吡唑环上的 N-NH 基序是抑制剂具有良好抗病 毒效力的关键结构特征, 在晶体结构中可观察到 N-NH 基序能与逆转录酶上的 K103 存在氢键作用(图 8) ${ }^{[37]}$.

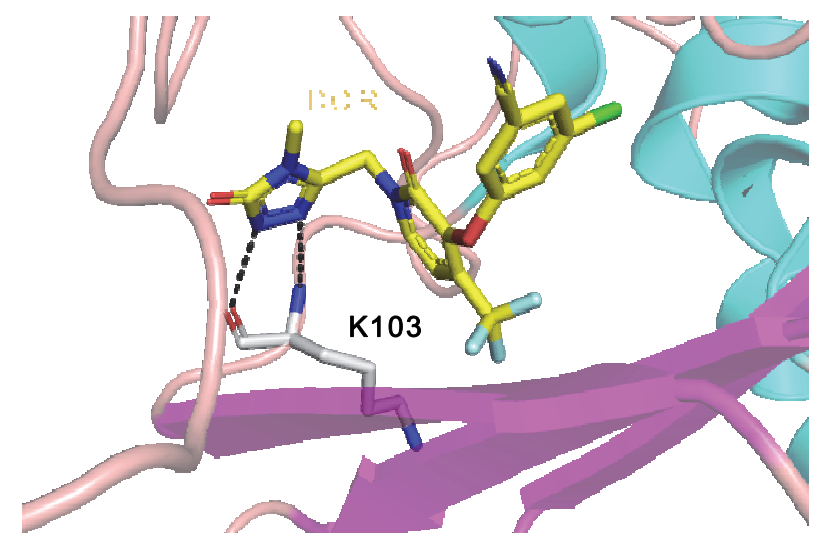

图 8 Doravirine 与逆转录酶复合物晶体结构 (PDB 号 $4 \mathrm{NCG})^{[37]}$

Figure 8 Crystal structure of Doravirine in complex with reverse transcriptase (PDB code $4 \mathrm{NCG})^{[37]}$

Hydrogen bonds are depicted by black dashed lines

2015 年默克公司 ${ }^{[38]}$ 通过流动化学开发了一种新的 Doravirine 合成方法(Scheme 3)：该方法利用连续的羟 


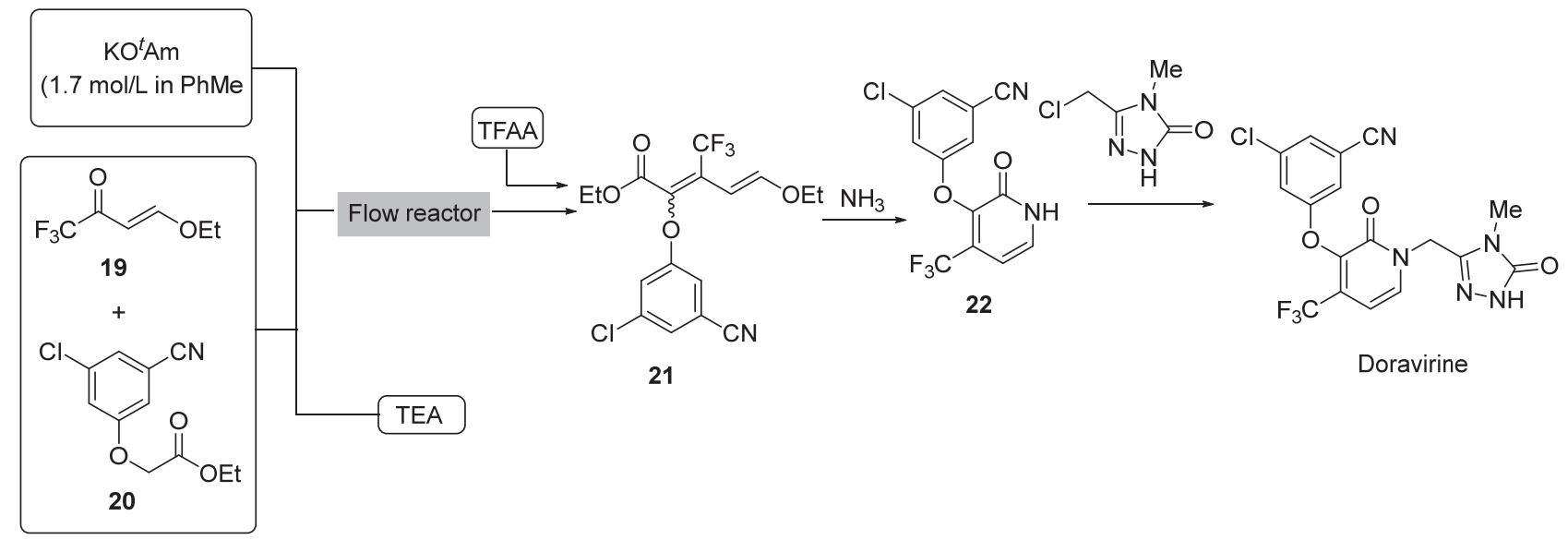

图式 3 Doravirine 的高效合成路线

Scheme 3 Highly efficient synthesis of doravirine

醛缩合反应将酯 20 稳定转化成吡啶酮 $\mathbf{2 2}$, 从而避免不 稳定的烯醇中间体形成. 最终在优化的流动条件中以 52\%的总收率和优异的化学纯度制备得到 Doravirine.

在体外实验中, Doravirine 对野生型、 K103N 和 $\mathrm{Y} 181 \mathrm{C}$ 耐药突变体的 $\mathrm{IC}_{50}$ 分别为 $12 、 9.7$ 和 $9.7 \mathrm{nmol} / \mathrm{L}$, 且在 50\%正常人血清中 Doravirine 表现出优异的抑制病 毒复制效果，其对野生型、K103N、Y181C 以及 K103N/ $\mathrm{Y} 181 \mathrm{C}$ 逆转录酶突变体的 $\mathrm{EC}_{95}$ (95\% effective concentration)为 20、43、27 和 $55 \mathrm{nmol} / \mathrm{L}$. Doravirine 对不同 HIV-1 亚型有着类似的抗病毒效力, 与其他 FDA 批准的 抗病毒药物联用未发现任何拮抗作用 ${ }^{[39]}$. 然而, Doravirine 在外周血单核细胞中对 HIV-2 的抑制效果较差 $\left(\mathrm{EC}_{50}=1.25 \mu \mathrm{mol} / \mathrm{L}\right)^{[40]}$. 在临床试验中 Doravirine 消除 半衰期为 $15 \mathrm{~h}$, 在体内主要通过 CYP3A 介导消除代 谢 ${ }^{[41]}$. 一系列 DRIVE 第三阶段临床试验证明 Doravirine 具有长效性且产生的不良反应处于可接受范围 ${ }^{[42]}$. Delstrigo 是口服固定剂量的三合一复方片剂, 由多拉维林 (Doravirine, DOR, $100 \mathrm{mg}$ )、拉米夫定(Lamivudine, 3TC, $300 \mathrm{mg}$ )和富马酸替诺福韦二吡呋酯(Tenofovir disoproxil fumarate, TDF, $300 \mathrm{mg}$ )组成. Delstrigo 复方片剂相比 于 Efavirenz/Emtricitabin/Tenofovir disoproxil fumarate $(600 / 200 / 300 \mathrm{mg})$ 复方制剂来说, 其无明显的中枢神经 系统不良反应且对脂代谢影响小 ${ }^{[43]}$. Doravirine 单剂治 疗和 Doravirine 联合治疗方案可用于无既往抗逆转录病 毒药物治疗史的 HIV-1 感染患者. 作为一线药物, Doravirine 能够对 NNRTIs 产生的经典耐药突变体 K103N, Y181C 和 G190A 具有抗病毒活性、良好的耐受性和吸 收性, 其不良反应处于可接受范围, 这些优点使它成为 一个极具吸引力的抗逆转录病药物.

\subsubsection{Elsulfavirine (VM1500)}

艾法韦林(Elsulfavirine, VM1500)是 Viriom 公司研
发的 NNRTIs 药物(图 9), 并于 2017 年 7 月在俄罗斯批 准上市. Elsulfavirine 是活性化合物 VM1500A 的前药, 以每日一次 $20 \mathrm{mg}$ 的口服胶囊供应 ${ }^{[44]}$. 在体外实验中 Elsulfavirine 具有纳摩尔级别的抗病毒活性 $\left(\mathrm{EC}_{50}=1.2\right.$ $\mathrm{nmol} / \mathrm{L}$ ), 对于 NNRTIs 产生的常见的耐药突变体具有抗 病毒活性 ${ }^{[45]}$. 在Ib/IIa 期临床试验中, Elsulfavirine 被证 明是安全有效的. 在之后的 IIb 期试验中, Elsulfavirine 与 Tenofovir disoproxil fumarate 和 Emtricitabin 联合使用 的安全性和有效性与 Efavirenz 相当, 并且服用 Elsulfavirine 组的不良反应概率为 $36.7 \%$, 明显低于 Efavirenz 组的 77.6\% ${ }^{[46]}$. 总而言之, Elsulfavirine 安全性 与耐受性明显好于 Efavirenz, 中枢神经系统不良反应发 生率低且无过敏现象 ${ }^{[4]}$. Viriom 公司还在开发一种肌内 长效可注射纳米制剂 VM-1500A-LAI，现已开始第二阶 段研究.



图 9 Elsulfavirine 化学结构

Figure 9 Chemical structure of Elsulfavirine

\section{3 蛋白酶抑制剂}

HIV 基因编码的天冬氨酰基蛋白酶是影响 HIV-1 基 因组复制的关键酶之一，它能在病毒复制晚期催化 Gap 和 Gap-Pol 前体多聚蛋白水解形成酶和结构蛋白(P17, P24，P9 和 P7), 从而使病毒具有感染性 ${ }^{[47]}$. 蛋白酶抑制 剂((PIs))能够抑制蛋白酶活性，从而产生不成熟且无感 染性的病毒颗粒, 达到抗病毒的效果. 如图 10 所示, 美 国 FDA 批准的 HIV 蛋白酶抑制剂有沙奎那韦 (Saquinavir, SQV)、荫地那韦(Indinavir, IDV)、利托那韦 (Ritonavir, RTV)、奈非那韦(Nelfinavir, NFV)、替拉那韦 


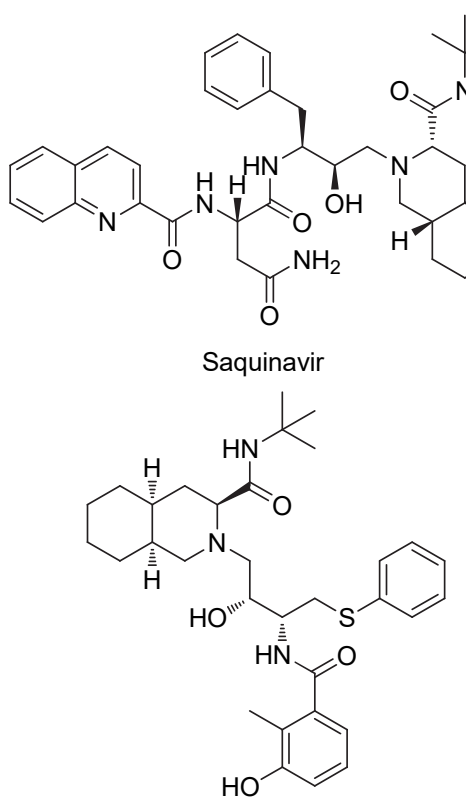

Nelfinavir<smiles>CC(C)(C)NC(=O)[C@H]1CN(Cc2cccnc2)CCN1CC(O)C[C@@H](Cc1ccccc1)C(=O)N[C@H]1c2ccccc2CC1O</smiles>

Indinavir<smiles>CCCC1(CCc2ccccc2)CC(O)=C([C@H](CC)c2cccc(NS(=O)(=O)c3ccc(C(F)(F)F)cn3)c2)C(=O)O1</smiles>

Tipranavir<smiles>CC(C)c1nc(C(C)N(C)C(=O)NC(C(=O)NC(Cc2ccccc2)CC(O)Cc2ccccc2)C(C)C)cs1</smiles><smiles>CC(C)CN(CC(O)[C@H](Cc1ccccc1)NC(=O)OCC1COC2OCCC12)S(=O)(=O)c1ccc(N)cc1</smiles>

Darunavir

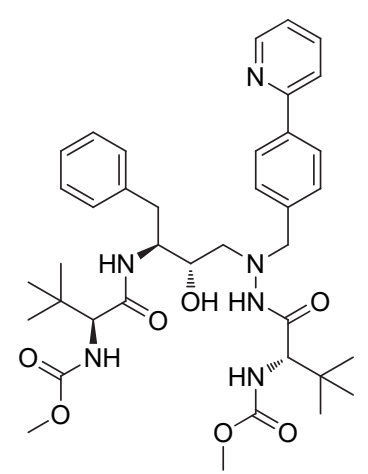

Atazanavir<smiles>CC(C)CN(C(=O)OC1CCOC1)C(OC(=O)NC(Cc1ccccc1)C(=O)O)C(=O)O</smiles>

Fosamprenavir<smiles>CC(C)CN(CC(O)C(Cc1ccccc1)NC(=O)OC1CCOC1)S(=O)(=O)c1ccc(N)cc1</smiles>

Amprenavir<smiles>Cc1cccc(C)c1OCC(=O)N[C@@H](Cc1ccccc1)[C@H](O)C[C@@H](Cc1ccccc1)NC(=O)[C@@H](C(C)C)N1CCCNC1=O</smiles>

Lopinavir

图 10 FDA 批准 PIs 药物

Figure 10 Structures of FDA-approved PIs

(Tipranavir, TPV)、达芦那韦(Darunavir, DRV)、阿扎那 韦(Atazanavir, ATV)、夫沙那韦(Fosamprenavir, FAPV)、 安瑞那韦 (Amprenavir, APV) 和洛匹那韦 (Lopinavir, LPV). HIV 蛋白酶抑制剂主要分为拟肽类药物和非肽类 药物, 如果用药不当, 可能会出现一些交叉耐药性问题.

\subsection{1 基于 DRV 结构修饰后 PIs}

达芦那韦(Darunavir, DRV)于 2006 年经 FDA 批准上 市, 与 Ritonavir 及其他抗逆转录病毒药物联合使用可治 疗 HIV-1 初治及经治患者(图 11a) 浮 $^{[4]}$. 经改良后的 Darunavir 联合 Cobicistat 的复合制剂(Darunavir/cobicistat, DRV/c)于 2013 年批准上市, 已得到广泛使 用 ${ }^{[49]}$. 由共晶结构可知, Darunavir 的双四氢呋喃环中的 2 个氧 均与蛋白酶活性位点 D29 和 D30 残基存在紧密的氢键 作用从而阻碍底物与酶结合, 竞争性抑制蛋白酶活 性 ${ }^{[50]}$ (图 11b). 同时也有研究表明 Darunavir 可以抑制蛋 白酶二聚化 ${ }^{[51]}$. Darunavir 抗病毒效果和安全性良好, 适 a<smiles>CCCN(CC(O)C(Cc1ccccc1)NC(=O)O[C@H]1CCO[C@@H]2OCC[C@@H]12)S(=O)(=O)c1ccc(N)cc1</smiles>

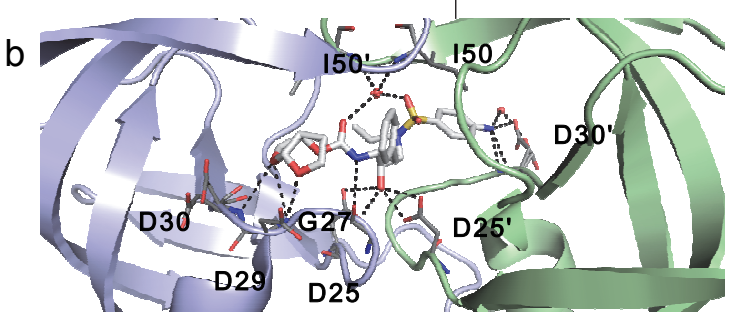

图 11 (a) Darunavir 化学结构和(b) Darunavir 与蛋白酶复合物 晶体结构(PDB 号 4LL3) ${ }^{[50]}$

Figure 11 (a) Structure of Darunavir and (b) crystal structure of Darunavir in complex with protease (PDB code 4LL3) ${ }^{[50]}$ Different proteases are coloured in cyan and light blue, respectively. Hydrogen bonds are depicted by black dashed lines 
用于与其他抗逆转录药物联合用药. 目前在 Darunavir 结构基础上进行化学修饰已经成为研究新型 HIV-1 的主 要方向.

Hohlfeld 等[52]在双四氢呋喃环(bis-THF)上 4-C 位引 入烷氧基、磺胺基及卤代烃衍生物等取代基, 得到的大 部分化合物在纳摩尔级浓度即可对 HIV-1 野生型及多药 耐药型产生有效的抑制作用. 化合物 23 中的甲氧基乙 氧基部分通过水分子介导与处于活性位点中发卡结构 附近的 G48 中的胺基发生氢键作用从而增强其抑制能 力(图 12a， 12b); 化合物 $\mathbf{2 4}$ 中的乙酰胺与蛋白酶中 G48 的羰基形成氢键(图 12c, 12d); 化合物 $\mathbf{2 5}$ 中的三氟甲基 结构可能与蛋白酶 G48 中的羰基氧存在相互作用并表 现出亚纳摩尔级的抑制作用(图 12e, 12f).

Agniswamy 等[53]发现在双四氢呋喃环 4-C 上引入两 个氟可以增加其脂溶性, 改善药物通过血脑屏障的能 力, 同时与 G48 的主链发生范德华作用. 除此之外, 其 他研究还在双四氢呋喃环上引入酯类、酰胺和脲等结
构, 或者将双四氢呋喃环改造成双环啞唑烷酩 ${ }^{[54]}$ 、四氢

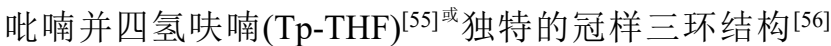
等.

\subsection{2 富勒烯类 PIs}

Strom 等[57]利用固相多肽合成法得到一系列富勒烯 $\mathrm{C}_{60}$ 衍生物, 研究表明其具有较好的 HIV 蛋白酶抑制活 性。通过定量构效关系(QSAR)分析了富勒烯衍生物的 结构-活性关系，结果表明富勒烯 $\mathrm{C}_{60}$ 能够被蛋白酶两条 链之间的直径约 $1.0 \mathrm{~nm}$ 圆柱形疏水口袋所容纳, 并且与 活性位点的非极性残基之间存在范德华作用力，从而影 响蛋白酶与底物结合 ${ }^{[58]}$.

2021 年 Yasuno 等 ${ }^{[59]}$ 合成三种类型的新型富勒烯衍 生物: 吡啶型衍生物 $26 \mathrm{a}$ 和 $27 \mathrm{~b}$ 、哌啶型衍生物 28 和脯 氨酸型衍生物 29a 31(图 13). 在化合物评估中发现 27a, 29e, 29f, 29i, 30a 30d 和 31 可以同时抑制 HIV 逆 转录酶和 $\mathrm{HIV}$ 蛋白酶, 且 $\mathrm{IC}_{50}$ 值处于低微摩尔范围. 由 于羟甲基羰基结构(Hydroxylmethylcarbonyl, HMC)能够

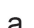<smiles></smiles>

b
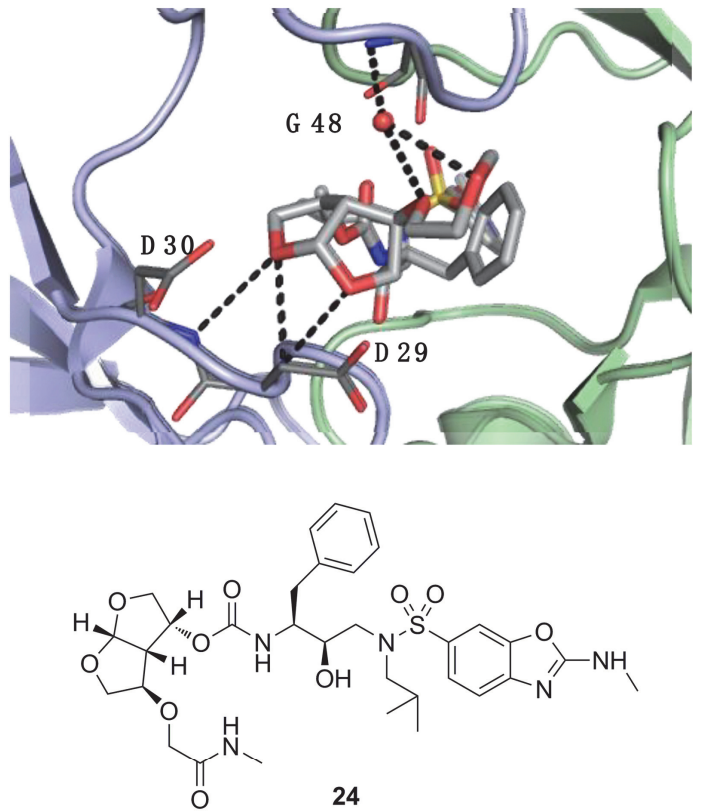

d

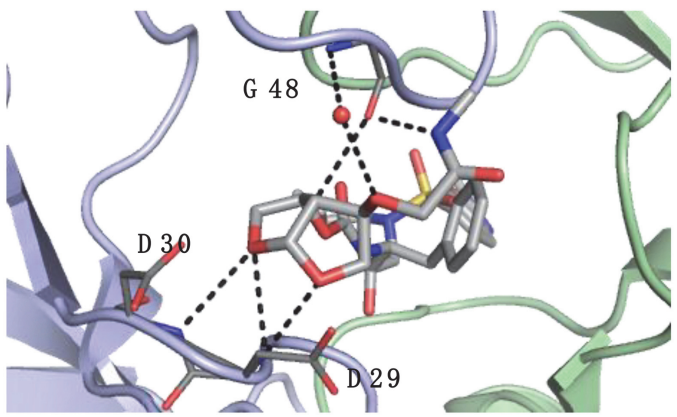

e
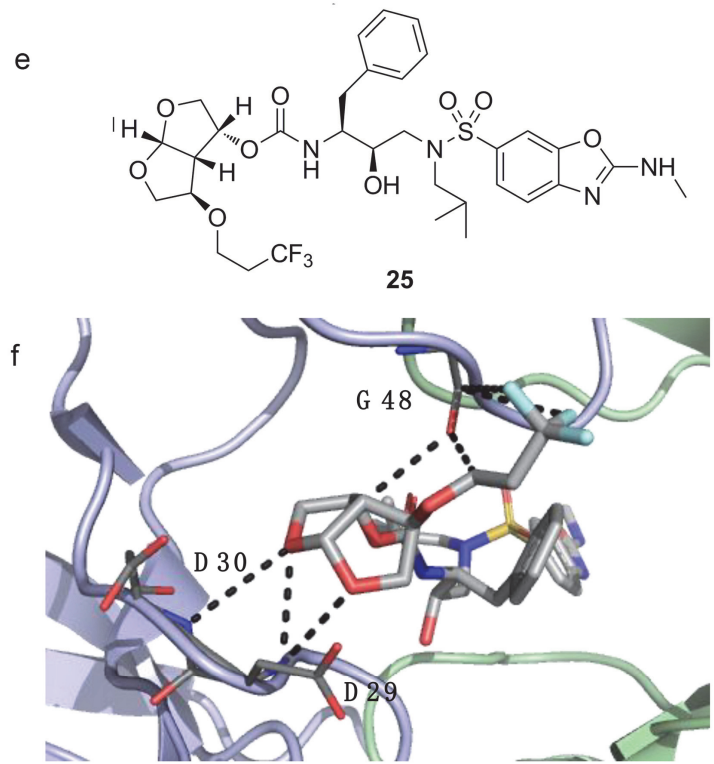

图 12 (a, c, e) 化学物 $23 \sim 25$ 结构和(b, d, f)化合物 $\mathbf{2 3} \sim \mathbf{2 5}$ 分别与蛋白酶复合物晶体结构(PDB 号 $5 \mathrm{AHA}, 5 \mathrm{AHB}, 5 \mathrm{AH} 8)^{[52]}$

Figure 12 (a, c, e) Chemical structures of compounds $\mathbf{2 3} \sim \mathbf{2 5}$ and (b, d, f) crystal structures of compounds $23 \sim \mathbf{2 5}$ in complex with protease, respectively (PDB code 5AHA, 5AHB, 5AH8) ${ }^{[52]}$

Different proteases are colored in cyan and light blue, respectively. Water molecule is indicated by magenta sphere, and hydrogen bonds are depicted by black dashed lines. 
与 HIV 蛋白酶活性位点的天冬氨酸残基发生相互作用, 故通过适当长度的烷基链将 $\mathrm{HMC}$ 结构与吡咯烷环连接 的脯氨酸型衍生物 30a 32d 和 31 具有更加优异的 HIV 蛋白酶抑制活性.
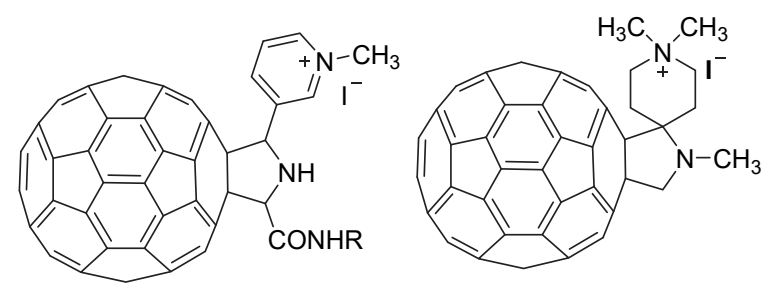

26a $\mathrm{R}=\mathrm{CH}_{3}$

26b $\mathrm{R}=\mathrm{C}_{2} \mathrm{H}_{5}$

27a $\mathrm{R}=\mathrm{OCH}_{3}$

27b $\mathrm{R}=\mathrm{OC}_{2} \mathrm{H}_{5}$

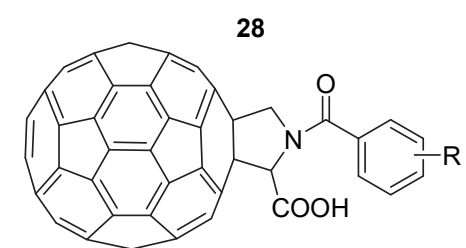

29a $\mathrm{R}=3-\mathrm{NHCOCH}_{2} \mathrm{OH}$

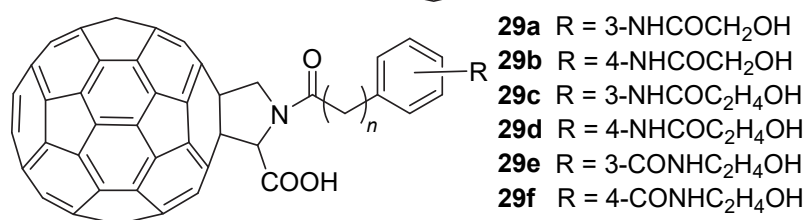

$\begin{array}{ll}\text { 30a } & \mathrm{R}=3-\mathrm{NHCOCH}_{2} \mathrm{OH}, n=1 \\ \text { 30b } & \mathrm{R}=4-\mathrm{NHCOCH}_{2} \mathrm{OH}, n=1 \\ \text { 30c } & \mathrm{R}=3-\mathrm{NHCOCH}_{2} \mathrm{OH}, n=2 \\ \text { 30d } & \mathrm{R}=4-\mathrm{NHCOCH}_{2} \mathrm{OH}, n=2\end{array}$



31

图 13 富勒烯类 HIV-1 蛋白酶抑制剂

Figure 13 Fullerene-based inhibitors of HIV-1 protease

\subsection{3 天然活化化合物}

Liu 等 ${ }^{[60]}$ 从斑点嗜蓝狍孔菌中得到了一种新的多糖 组分 $\mathrm{G} 1$, 由阿拉伯糖、果糖、半乳糖和葡萄糖组成. G1 具有显著的抗氧化作用, 在 $50 \mu \mathrm{g} / \mathrm{mL}$ 浓度时对 HIV-1 蛋白酶抑制作用约 $19.6 \%$, 同时在 $100 \sim 1000 \mu \mathrm{g} / \mathrm{mL}$ 浓 度时对 HIV-1 整合酶也具有抑制作用.

Chen 等 $\left[{ }^{61]}\right.$ 从链霉菌 sp. CPCC 中分离得到了一种线 性肽 Ahmpatinin ${ }^{i} \mathrm{Bu}$ (图 14), 其对 HIV 蛋白酶具有显著 抑制效果 $\left(\mathrm{IC}_{50}=1.79 \mathrm{nmol} / \mathrm{L}\right)$, 可以作为一种新型的 HIV 蛋白酶抑制剂进行后续研究.<smiles>COc1ccc(CC(NC(=O)C(C)NC(=O)CC(O)C(CC(=O)O)NC(=O)C(NC(=O)C(NC(=O)C(C)C)C(C)C)C(C)C)C(C)C)cc1</smiles>

图 14 Ahmpatinin ${ }^{i} \mathrm{Bu}$ 化学结构

Figure 14 Chemical sructure of Ahmpatinin ${ }^{i} \mathrm{Bu}$

\section{4 整合酶抑制剂}

在 HIV 复制过程中, 整合酶能与病毒 DNA 形成复 合物并通过 $3^{\prime}$ 末端加工(3'EP)和链转移过程完成将病毒 基因组整合入宿主染色体 ${ }^{[62]}$. INSTIs 药物因具有高效低 毒及药物相互作用小等优点成为 ART 治疗的热门药物. 如图 15 所示, FDA 批准的 INSTIs 药物有拉替拉韦 (Raltegravir, RAL)、艾维雷韦(Elvitegravir, EVG)、多替 拉维(Dolutegravir, DTG)、比卡格韦(Bictegravir, BIC)和 卡博特韦(Cabotegravir, CAB).<smiles>[R16]CC(C)(C)c1nc(C(=O)NCc2ccc(C)cc2)c(O)c(=O)n1C</smiles>



图 15 FDA 批准 INSTIs 药物

Figure 15 Structures of FDA-approved INSTIs

长效整合酶抑制剂(INSTIs)卡博特韦(Cabotegravir, CAB，GSK1265744)是 ViiV Healthcare 公司研制的新一 代整合酶抑制剂. 2021 年 1 月 22 日, Cabotegravir 与 Rilpivirine 联合缓释注射剂 Cabenuva 获得 FDA 批准上 市, 作为首个长效 HIV 组合疗法, 它意味着患者由 365 天全天接受治疗转变为每月一次接受治疗便可达到抑 制病毒效果. 同时, FDA 也批准了 Cabotegravir 口服片 剂 Vocabria 的上市.

Cabotegravir 与 Dolutegravir 具有相似化学结构(图 15). Cabotegravir 的合成主要面临两个挑战: 高度功能 化的吡啶酮核心构建以及噁唑烷部分的立体选择性. 葛 兰素史克公司开发了一种有效且具有高非对映选择性 的 Cabotegravir 合成方法(Scheme 4) ${ }^{[63]}$. 通过四步骤一 锅化反应以 $61 \%$ 的产率构建出含吡啶酮核心的中间体 35, 35 与 2,4-二氟芐胺偶联后再通过缩醛脱保护得到醛 36. 整合酶通过活性位点 Asp64、Asp116、Glu152 结合 $1 \sim 2$ 种金属阳离子以催化链转移过程，而抑制剂则通 过与金属离子螯合从而阻碍金属离子与整合酶催化位 
<smiles>COCC(=O)CC(=O)OC</smiles>

$61 \%$ yield over 4 steps

(1) CDI, then DFBA

(2) $\mathrm{HCOOH}$

$90 \%$ yield over 2 steps<smiles>COC(=O)c1c(OC)c(=O)c(C(=O)NCc2ccc(F)cc2F)cn1CC=O</smiles>

\section{(1) I-alaninol, $\mathrm{Mg}(\mathrm{OTf})_{2}$ $\mathrm{CH}_{3} \mathrm{CN}, 60^{\circ} \mathrm{C}$ \\ (2) $\mathrm{NaBr}$ \\ $52 \%$ yield over 2 steps \\ $(297: 1 d r)$}



Cabotegravir

图式 4 非对映选择性合成 Cabotegravir ${ }^{[63]}$

Scheme 4 Diastereoselective synthesis of Cabotegravir ${ }^{[63]}$

点结合. 通常 $\mathrm{Mg}^{2+}$ 离子被认为是参与整合过程的金属 辅助因子, 故整合酶抑制剂一般被设计成 $\mathrm{Mg}^{2+}$ 螯合剂, 并且 Cabotegravir 的螯合位点为 5-C、6-C 和 7-C 区域. 同 时化合物 36 对 $\mathrm{Mg}^{2+}$ 离子的鳌合作用对于噁唑烷部分的 闭环反应的非对映选择性产生重大影响, 故利用 $\mathrm{Mg}^{2+}$ 离子介导闭环反应可以生成更稳定的过渡态并且以极 高的非对映选择性 $(d r=297:$ 1)合成 Cabotegravir.

Cabotegravir 表现出极其优异的抑制作用: 在体外 实验中, Cabotegravir 对外周血单核细胞中的 HIV-1 BAL 和 NL432 的 $\mathrm{IC}_{50}$ 分别为 0.22 和 $0.34 \mathrm{nmol} / \mathrm{L}$; 在体内实 验中, Cabotegravir 与血清白蛋白高度结合, $\mathrm{IC}_{90}$ 仅为 166 $\mathrm{ng} / \mathrm{mL}^{[64]}$. Cabotegravir 在皮下或肌内注射吸收迅速, 一 次性口服 $30 \mathrm{mg}$ Cabotegravir, 药物半衰期可达 $40 \mathrm{~h}^{[65]}$. Cabotegravir 良好的耐受性、安全性以及其药代动力学 特征使之能够被制成每日一次的口服片剂和每月或每 季度给药的长效纳米悬浮剂. 因其水溶性差, 故将 Cabotegravir 制成纳米混悬针剂后可明显改善其溶解性 和吸收能力 ${ }^{[64]}$. 长效 Cabotegravir 纳米混悬针剂含有 $200 \mathrm{mg} / \mathrm{mL}$ 的结晶活性药物, 平均粒径为 $200 \mathrm{~nm}$, 半衰 期可达到 21 50 $\mathrm{d}^{[66]}$. Cabotegravir/Rilpivirine 长效药物 联合制成的可临床使用的纳米悬液针剂, 通过两个III期 临床试验 ATLAS 和 FLAIR 的研究结果证明每月一次注 射该针剂便可长效抑制病毒复制, 疗效明显且依从性和 耐受性良好 ${ }^{[67]}$. 为了探究 Cabotegravir 在 PrEP 中的应用 潜力, 将 Cabotegravir 作为预防剂后将恒河猴阴道反复 暴露于 SHIV 162P3, 此研究表明 Cabotegravir 能使猴子 阴道免于感染 SHIV ${ }^{[68]}$. 综上所述, Cabotegravir 在 HIV-1 感染的治疗以及预防方面有着良好的应用前景, 能够明 显提高病人依从性.

\section{5 侵入抑制剂}

HIV-1 的 env 基因可编码约 863 个氨基酸的前体蛋 白, 后者糖基化形成 gp160 前体, gp160 在蛋白酶催化作 用下裂解为表面糖蛋白 gp 120 和跨膜糖蛋白 gp41. 成熟 HIV 病毒粒子上的 gp120 与 gp41 三聚体可介导病毒与 宿主细胞结合. 首先, gp120 与宿主细胞的 CD4 受体结 合, 导致 gp120发生相对的空间移动和构型变化; 随后, gp120 与趋化因子受体(CCR5 或者 CXCR4)结合暴露出 gp41; 最后, gp41 改变构型形成 6 股 $\alpha$ 螺旋束核心结构 (6-HB), 固定融合孔从而介导病毒与宿主细胞膜融 合 ${ }^{[69-70]}$. HIV 侵入抑制剂能抑制病毒进入靶细胞, 从而 在最早阶段抑制病毒传播. 根据病毒侵入宿主细胞不同 步骤, 可以将侵入抑制剂分为附着抑制剂(Attachment inhibitors)、附着后抑制剂(Post-attachment inhibitors)、 趋化因子受体抑制剂(Chemokine receptor inhibitors)以 及融合抑制剂(Fusion inhibitors). 根据其不同理化性质, 侵入抑制剂也可分为蛋白多肽类、中和抗体类和有机小 分子.

\subsection{1 附着抑制剂}

附着抑制剂能够干扰病毒表面糖蛋白 gp120 与 CD4 受体结合从而阻止病毒附着靶细胞. Fostemsavir (FTR, GSK-3684394, BMS-663068)是一种由百时美施贵宝研 研发现并被 ViiV Healthcare 收购的附着抑制剂, 是 Temsavir (BMS-626529)的一种研究性前药(图 16a). 2020 年 7 月 3 日 FDA 批准上市的 Rukobia (fostemsavir) 600 $\mathrm{mg}$ 口服片剂可用于联合其他逆转录病毒药物治疗多重 耐药 HIV 成人感染者 ${ }^{[71]}$. Fostemsavir 与表面糖蛋白 gp120 结合而防止 HIV 病毒附着在 CD4 受体上, 进而防 止 HIV 感染宿主细胞. Temsavir 与 gp120 的共结晶结 构 ${ }^{72]}$ 显示 Temsavir 主要通过疏水作用与 gp120 结合, 并 
且与 D113 和 W427 残基存在氢键作用(图 16b). 当 Temsavir 与 gp120 结合后, 小分子的苯甲酰胺结构占据 着原先 W427 的位置从而将 W427 和 $\beta 20-\beta 21$ 环推向 CD4 受体结合位置, 阻碍 gp120 与 CD4 受体结合 ${ }^{[73]}$.

a<smiles>COc1cnc(-n2cnc(C)n2)c2c1c(C(=O)C(=O)N1CCN(C(=O)c3ccccc3)CC1)cn2COP(=O)([O-])O</smiles>

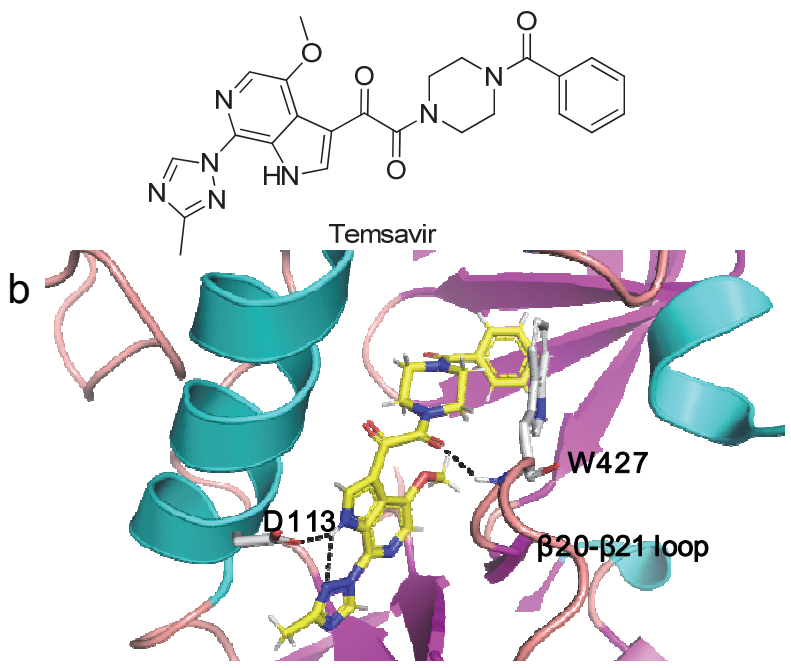

图 16 (a) Temsavir 和 Fostemsavir 化学结构和(b) Temsavir 与 gp120 复合物晶体结构(PDB 号 $5 \mathrm{U} 7 \mathrm{O})^{[72]}$

Figure 16 (a) Chemical structures of Temsavir and Fostemsavir and (b) crystal structure of Temsavir in complex with gp120 $(\mathrm{PDB} \text { code } 5 \mathrm{U} 7 \mathrm{O})^{[72]}$

Hydrogen bonds are depicted by black dashed lines

在体外实验中, Temsavir 具有较小的细胞毒性, 对 于多数病毒分离株而言, 其 $\mathrm{EC}_{50}$ 低于 $10 \mathrm{nmol} / \mathrm{L}$, 表明 其对大部分 HIV-1 亚型均有抗病毒作用 ${ }^{[74]}$. 在 HIV 多重 耐药患者中开展的关键性 III 期临床研究 BRIGHT (NCT02362503) 用以评估 Fostemsavir 的安全性及有效 性. 在该研究的 96 周结果显示前 24 周内病毒载量显著 减少, 从 24 到 96 周的病毒学抑制和免疫应答率继续增 加. 常见不良事件包括恶心、呕吐、腹泻、疲劳和头 痛 ${ }^{[75]}$. Rukobia (Fostemsavir) $600 \mathrm{mg}$ 缓释片剂可以每 12 $\mathrm{h}$ 口服一次, 为难以治疗的多重耐药患者提供了一种全 新的治疗方案.

\subsection{2 附着后抑制剂}

Tai Med 生物公司研发的 Trogarzo (ibalizumab-uiyk) 于 2018 年 3 月由 FDA 批准上市. Trogarzo 是一种静脉 滴注的人源化免疫球蛋白 $\mathrm{G} 4$ 单克隆抗体, 开发用于多
药耐药性 1 型人类免疫缺陷病毒感染的治疗 ${ }^{[76]}$. 一般情 况下, gp120 与 CD4 受体结构域 1 结合后发生构象变化 使 gap120 三聚体从封闭状态转换为开放状态 ${ }^{[77]}$. ibalizumab 具有全新作用机制: 它对人体 CD4 受体结构域 2 的 $\mathrm{N}$ 端有独特专一性的结合能力, 并不影响 gp120 与 CD4 受体结构域 1 结合, 但 ibalizumab 结合后能够引起 空间位阻，从而阻止 CD4 受体与 gp120复合物中的构象 变化, 使 gp120 不能与趋化因子受体相互作用进而阻止 病毒进入宿主细胞 ${ }^{[78]}$. 在 ibalizumab 的抗原结合片段 (Antigen-binding fragment, Fab)与 CD4 受体共晶结构中, ibalizumab Fab 段直接与 CD4 受体结构域 2 上的 B/C 环 (残基 121 125)结合并在此形成广泛的氢键网络(图 $17)^{[79]}$.

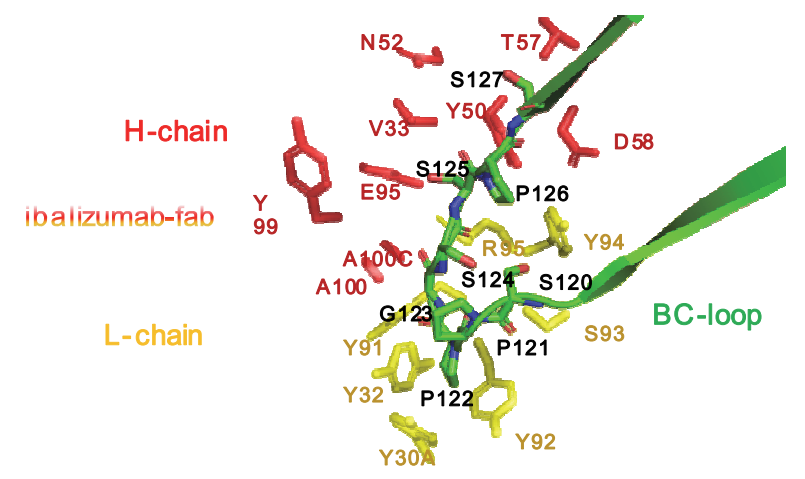

图 17 ibalizumab Fab 段与 CD4 受体结构域 2 复合物晶体结 构(PDB 号 3O2D) ${ }^{[79]}$

Figure 17 Crystal structure of the complex of 2 domain CD4 and the Fab fragment of ibalizumab (PDB code 3O2D) ${ }^{[79]}$

The heavy and light chains of ibalizumab are shown by surface representation in red and yellow, respectively, and the residues that make contacts with CD4 are also shown by stick model in the same color scheme. CD4 is in green in ribbon diagram and the residues interacting with ibalizumab are shown by stick model in green.

与其他广泛中和抗 HIV-1 单抗相比, ibalizumab 在 大多数 HIV 病毒株感染中具有优异的抑制效果，表明其 具有更好的抗病毒效力 ${ }^{[80]}$. 在 2018 年 ibalizumab 联合优 化背景疗法的III期临床试验 TMB-301 中，40 名感染多 重耐药性 HIV 的患者接受两周一次 ibalizumab 注射后, 治疗一周大部分患者血液中的 HIV-RNA 水平显著下降, 治疗 24 周 $43 \%$ 的患者 HIV-RNA 拷贝数 $<50$ 拷贝 $/ \mathrm{mL}$, $50 \%$ 的患者 HIV-RNA 拷贝数 $<200$ 拷贝 $/ \mathrm{mL}^{[81]}$. Trogarzo 作为一种创新疗法, 每 $14 \mathrm{~d}$ 注射一次提高了病人的依从 性, 并可与其他抗逆转录病毒药物联用降低耐药风险 ${ }^{[82]}$.

\subsection{3 趋化因子受体抑制剂}

在病毒侵入靶细胞过程中, 趋化因子受体 CCR5 或 CXCR4 的 $\mathrm{N}$ 末端酪氨酸和其它酸性氨基酸与 gp120 结 合从而导致 gp41 构型变化 ${ }^{[83]}$. 如图 18a 所示, 目前 FDA 批准的 CCR5 抑制类药物仅有马拉维若(Maraviroc, 
MVC). Maraviroc 通过氢键和盐桥与 CCR5 受体结合, 从而阻断 gp120 与 CCR5 受体相互作用(图 18b), 有效阻 止病毒进入细胞, 且可与其他抗逆转录病毒药物联用于 治疗 HIV-1 感染 ${ }^{[84]}$.

a

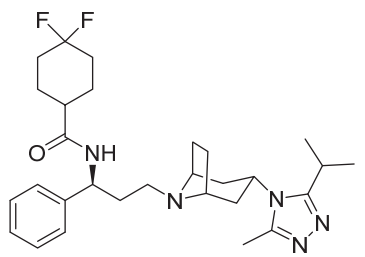

b

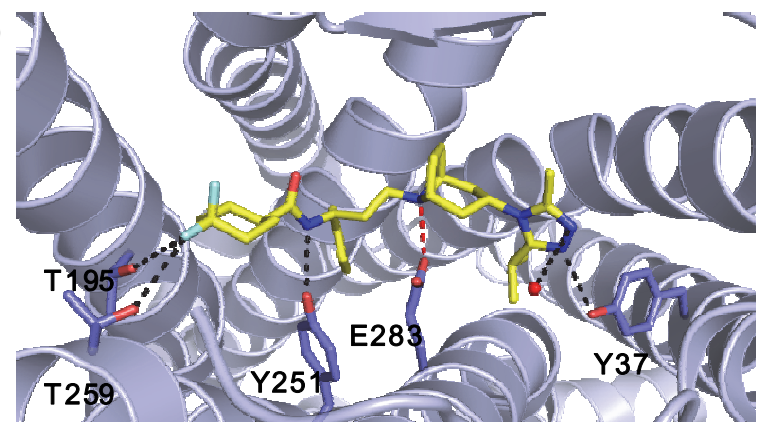

图 18 (a) Maraviroc 化学结构和(b) Maraviroc 与 CCR5 趋化因 子受体复合物晶体结构域(PDB 号 $4 \mathrm{MBS})^{[85]}$

Figure 18 (a) Structure of Maraviroc and (b) crystal structure of Maraviroc in complex with CCR5 chemokine receptor (PDB code $4 \mathrm{MBS})^{[85]}$

Hydrogen bonds and salt bridge are depicted by black and red dashed lines, respectively

手性药物 Maraviroc 的不对称合成引起了极大的关 注. 2019 年 Zhu 等 ${ }^{[86]}$ 开发了一种新颖有效的 Maraviroc 不对称合成途径(Scheme 5): 利用 Ellman 开发的(S)-叔 丁基亚磺酰胺作为手性辅助试剂制备手性胺, 总收率为 $34 \%$, 化学纯度为 $99.71 \%$, 手性纯度为 $99.94 \%$. 新路线
步骤简洁，反应条件温和且易于操作.

塞拉维诺(Thioraviroc) ${ }^{[87-88]}$ 是由中国科学院上海药 物研究所和中国科学院昆明动物研究所合作研发的新 型 CCR5 拮抗剂(图 19a), 现处于临床I期试验中. 基于 Maraviroc 与 CCR5 受体结合的晶体结构发现其酰胺部 分以及三坐基团对 CCR5 受体结合非常重要; 此外，在 对 Maraviroc 的代谢途径分析中得到苯基、酰胺部分以 及三唑基团是主要代谢位点. 为了提高抑制剂对 CCR5 的结合能力并提高代谢稳定性，设计了一系列新型

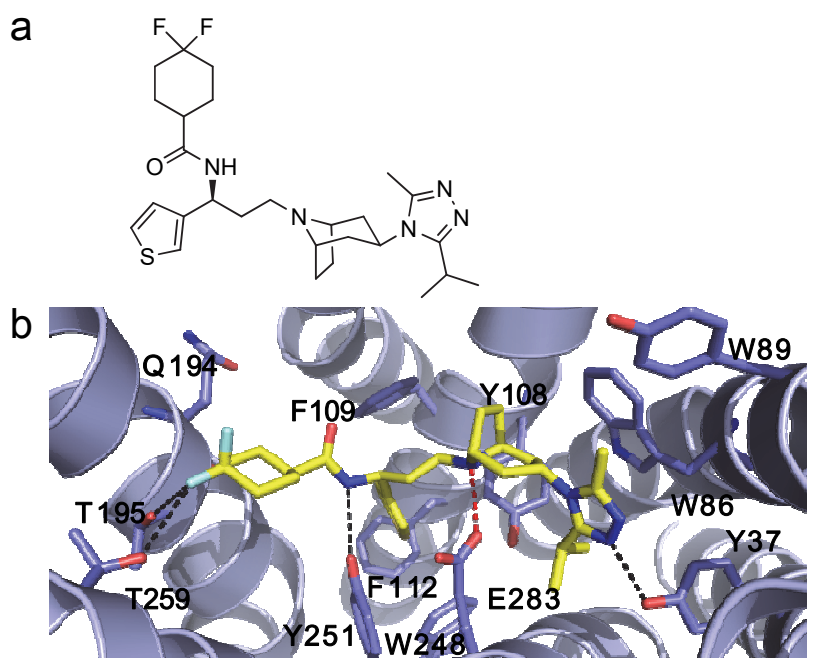

图 19 (a) Thioraviroc 化学结构和(b) Thioraviroc 与 CCR5 趋化 因子受体复合物晶体结构域(PDB 号 $6 \mathrm{AKY})^{[87]}$

Figure 19 (a) Chemical structure of Thioraviroc and (b) crystal structure of Thioraviroc in complex with CCR5 chemokine receptor (PDB code $6 \mathrm{AKY})^{[87]}$

Hydrogen bonds and salt bridge are depicted by black and red dashed lines, respectively

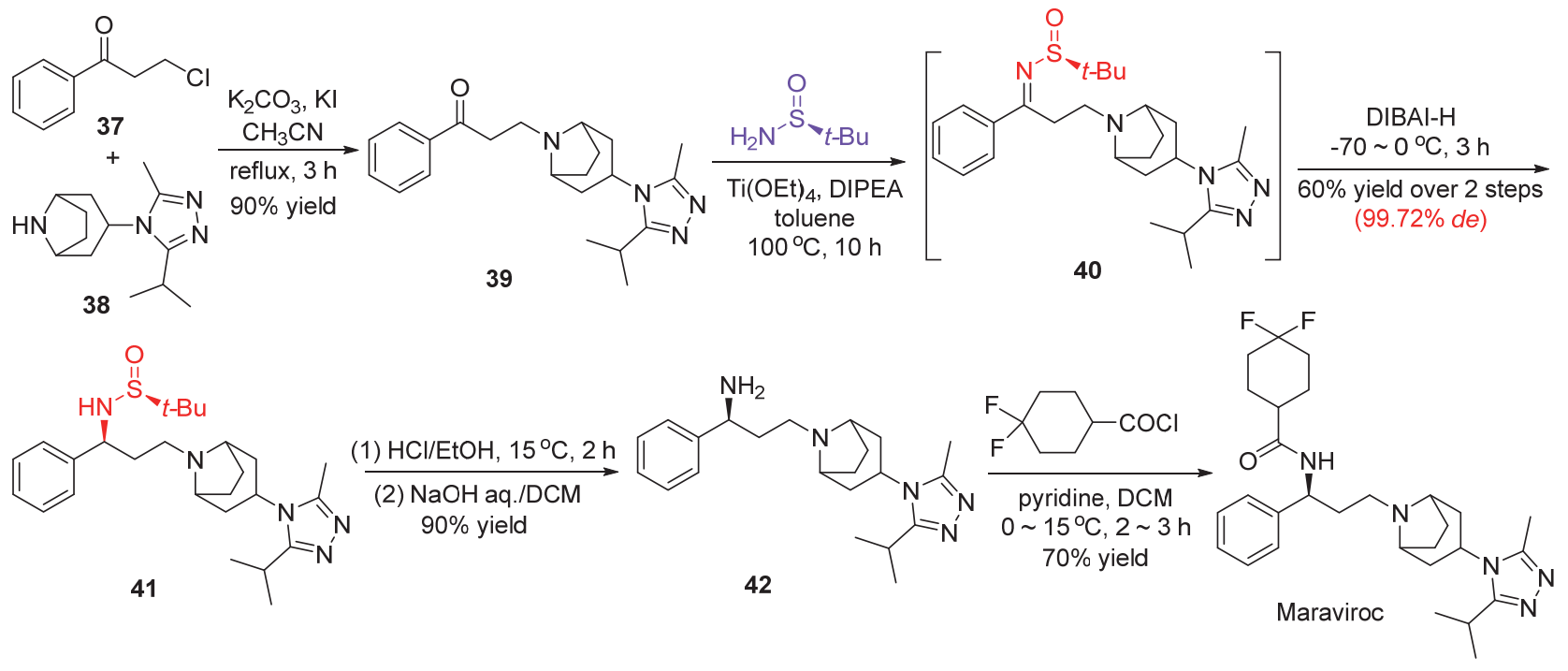

图式 5 Maraviroc 合成过程

Scheme 5 Synthetic process for Maraviroc 
CCR5 拮抗剂, 在多轮结构修饰后对其进行药效评价和 成药性优化. 最终得到 Thioraviroc, 其保留了酰胺基团 和三唑基团, 并用噻吩基团取代了苯基. 在共结晶结构 中可见噻吩部分深入到结合口袋, 与五个芳香族残基 Y108、F109、F112、W248 和 Y251 形成疏水相互作用; 环已烷上其中一个氟原子与 T195、T259 形成氢键(图 19b).

在体外实验中, Thioraviroc 抗 HIV-1 活性明显优于 Maraviroc, 且在低纳摩尔浓度下具有低细胞毒性. 塞拉 维诺具有良好的药代动力学特性, 与 Maraviroc 相比, 其显示出更好的代谢稳定性以及生物利用度. Thioraviroc 在 $25 \mu \mathrm{mol} / \mathrm{L}$ 时对 CYP450 酶无抑制作用, 无潜在药 物相互作用, 可成为治疗 HIV 感染的候选药物 ${ }^{[87]}$.

\subsection{4 融合抑制剂}

gp41 分为膜外区域、跨膜区域以及胞质区域. 膜外 区域包括融合肽(FP), $\mathrm{N}$ 末端重复序列(NHR), $\mathrm{C}$ 末端重 复序列 (CHR) 以及色氨酸丰富区 $(T R)$ 直接参与病毒融 合. 3 个 CHRs 通过反向平行并联的方式结合于 3 个 NHRs 表面, 形成稳定的 6-HB 拉近病毒与宿主细胞之


抗病毒融合抑制剂恩夫韦地(Enfuvirtide, T-20)是一条线 形的含有 14 种不同 L 型天然氨基酸的 36 肽(图 20), 其

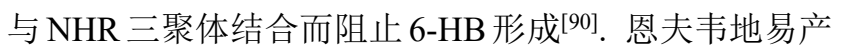
生耐药性且半衰期短 $(3.46 \sim 4.35 \mathrm{~h})$, 患者需一天皮下注 射两次, 故未获得广泛推广[91].

\section{$\mathrm{CH}_{3} \mathrm{CO}$-YTSLIHSLIEESQNQQEKNEQELLELDKWASLWNWF-NH ${ }_{2}$}

图 $20 \mathrm{~T}-20$ 结构

Figure 20 Structure of T-20

艾博卫泰(Albuvirtide, $\mathrm{ABT}$ )是我国自主研发的首个 长效抗 HIV 融合抑制剂, 已于 2018 年 6 月在国内上市. Albuvirtide 是以 C34 序列为模板设计的 3-马来酰亚胺丙 酸(3-maleimimidopropionic acid, MPA)修饰的多肽(图 21). Albuvirtide 属于针对 gp41 的融合抑制剂, 能够阻断 6-HB 的形成从而抑制病毒进入细胞. 人血清白蛋白 (Human serum albumin, HBS) 是改善药物动力学特征和 靶向给药的理想载体. 3-马来酰亚胺丙酸修饰的支链结 构可以迅速地与血清白蛋白形成不可逆的结合, 复合物 可以在血液中有效并密集地运输, 从而在体内实现长效
的血药浓度, 半衰期可达 $11 \sim 12 \mathrm{~d}$. Albuvirtide 具有良好 的水溶性、稳定性和低细胞毒性, 且对多种 HIV-1 亚型 和突变体均具有抑制活性 ${ }^{[92]}$. 在 Albuvirtide 与 lopinavir/ ritonavir (LPV/r) 两药联合使用研究中, 其表现出强大的 抗 HIV 活性、更好的安全性及更少的药物相互作用 [93]. 在III期临床试验 TALENT 中, 将一线抗逆转录药物治疗 失败的 HIV-1 感染者随机分为两组, 一组接受简化方案 (Albuvirtide $+\mathrm{LPV} / \mathrm{r}$ ), 另一组接受标准三联方案 (LPV/ $\mathrm{r}+$ Tenofovir disproxil fumarate/Zidovudine + Lamivudine); 48 周后, 简化方案组 $80.4 \%$ 的受试者 HIV RNA 拷贝数 $<50$ 拷贝 $/ \mathrm{mL}$, 三联方案 $66 \%$ 受试者 HIV RNA 拷贝数 $<50$ 拷贝 $/ \mathrm{mL}$. 该研究表明每周注射一次的 Albuvirtide 联合 $\mathrm{LPV} / \mathrm{r}$ 的治疗方案与世界卫生组织推荐的三联方案 疗效相当或更优, 且耐受性良好 ${ }^{[94]}$.

\section{6 其他新型制剂}

HIV 衣壳蛋白(Capsid protein, CA)在病毒复制的多 个阶段中有着关键作用, 常常被认为是治疗的关键靶点 之一. $g a g$ 基因表达的前体蛋白在蛋白酶的作用下被切 割成多种蛋白, 其中之一就是衣壳蛋白, 病毒将 250 个 $\mathrm{CA}$ 六聚体以及 12 个五聚体自发组装成雉形衣壳, 随后 衣壳将 HIV 基因组和酶完整包裹在一起完成病毒的复 制[95]. 2020 年 7 月吉利德公司报道了衣壳抑制剂 GS-6207 (Lenacapavir, LEN)在体外实验以及初步临床 试验中具有治疗 HIV 感染的长效潜力 ${ }^{[96]}$. 基于 PF3450074 (PF74)结构, 从 4000 个化合物中篎选出 GS-CA1, 逐步改造优化得到 GS-6207(图 22a). 在晶体 结构(图 22b)中可以看到 G2-6207 结合于 CA 六聚体其 中一个单体的 $\mathrm{N}$ 端以及相邻单体的 $\mathrm{C}$ 端之间的保守界 面处，且小分子与 $\mathrm{CA}$ 单体结合口袋有着显著的形状互 补性，两者通过广泛的疏水作用和静电作用紧密结合从 而使衣壳完整性受到破坏. GS-6207 在受感染的 MT-4 细 胞中表现出比 FDA 已批准的抗逆转录药物更加优越的 抗病毒效力 $\left(\mathrm{EC}_{50}=105 \mathrm{pmol} / \mathrm{L}\right)$. 在 HIV-1不同分离株中 展现其良好抑制作用，且对 HIV-2 也有一定抑制作用. 同时 GS-6207 的肝清除率低，这是成为长效抑制剂的基 础. 在I期临床试验中, 在感染 HIV-1 但未接受治疗的患 者中单剂量皮下注射 GS-6207 (20 450 mg), 药物安全 且耐受性良好, $450 \mathrm{mg}$ 剂量组 $9 \mathrm{~d}$ 后血浆中病毒载量<smiles>CCOCCNCCOCCOCCOCCNC(=O)NCCCN1C(=O)C=CC1=O</smiles>

图 $21 \mathrm{ABT}$ 结构

Figure 21 Sructure of ABT 

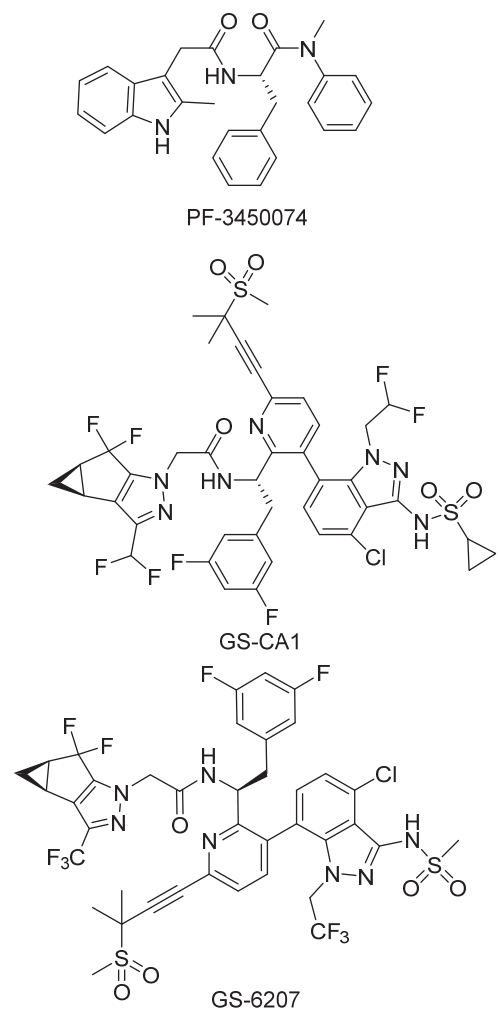

GS-6207



图 22 (a) PF-3450074, GS-CA1 以及 GS-6207 化学结构和(b) GS-6207 与 CA 六聚体复合物晶体结构(PDB 号 6V2F) ${ }^{[96]}$

Figure 22 (a) Chemcial structures of PF-3450074, GS-CA1 and GS-6207 and (b) crystal structure of GS-6207-CA hexamer complex (PDB code 6V2F) ${ }^{[96]}$

CA monomers are coloured alternately in cyan and light blue, hydrogen bonds and cation- $\pi$ interactions are depicted by black and red dashed lines, respectively.

明显降低且其有效抗病毒活性能够维持六个月以上. GS-6207 作为 HIV-1 衣壳抑制剂, 有着极强的抗病毒活 性, 安全性高, 半衰期长, 释药缓慢, 可以作为长期治 疗的理想选择.

成熟抑制剂(Maturation inhibitors, MIs)通过与 gag 前体蛋白结合干扰蛋白酶切割, 导致无法形成结构性蛋 白从而抑制病毒粒子成熟[97]. GSK2838232 是一种在研 的口服第 2 代 MIs(图 23), 在体外实验中显示出对 HIV-1 亚型良好的抗病毒活性. 在 I 期临床试验中观察到加入
Ritonavir (RTV)后, 其半衰期增加一倍, 可达 $34 \mathrm{~h}^{[98]}$. 在 IIa 期临床试验中评估了 GSK2838232 与增强剂 Cobicistat 联用后的安全性、药代动力学以及抗病毒活 性：感染 HIV 但未接受治疗的患者每日口服 $200 \mathrm{mg}, 10$ $\mathrm{d}$ 后 HIV-1 RNA 平均下降 $1.7 \log _{10}$ 拷贝 $/ \mathrm{mL}$ ，且未发现 严重不良反应 ${ }^{[99]}$.

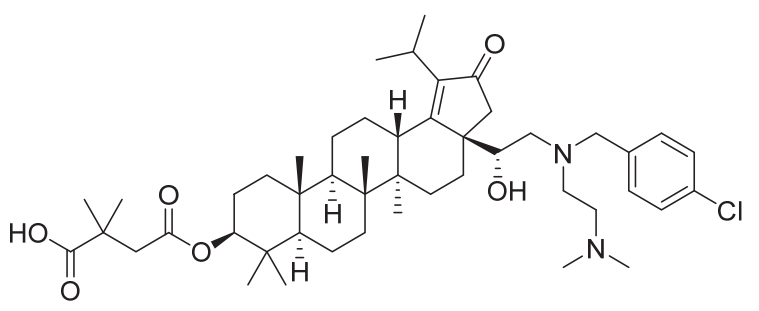

图 23 GSK2838232 化学结构

Figure 23 Chemical sructure of GSK2838232

\section{2 艾滋病其他治疗方式}

\subsection{HIV 疫苗}

开发一种安全有效 HIV 疫苗是控制和预防 HIV 传 播的重要策略. 三十年来, 数百个 HIV-1 疫苗已经过临 床试验测试，但至今没有一种完全有效且安全经济的 HIV 疫苗. 艾滋病疫苗的研发过程一般可分为三个阶 段：第一阶段，诱导体液免疫产生中和抗体；第二阶段， 诱导 $\mathrm{CD} 8^{+} \mathrm{T}$ 细胞介导的细胞免疫; 第三阶段, 诱导体液 免疫和细胞免疫两者结合. 目前艾滋病疫苗的种类包括 传统疫苗(灭活疫苗和减毒活疫苗)和新型疫苗(病毒样 颗粒、合成多肽疫苗、蛋白亚单位疫苗、脱氧核糖核酸 疫苗和载体疫苗). 其中, 重组病毒载体是 HIV-1 疫苗中 最具吸引力的载体工具. 彻底清除艾滋病的道路充满荆 䊂, 但我们相信不久的将来安全且有效的 HIV 疫苗将会 问世 ${ }^{[100]}$.

\subsection{HIV 的 CRISPR/Cas9 基因编辑}

从 ZFNs, TALENs 到 CRISPR/Cas9, 经历了三代技 术变革, CRISPR/Cas9 因其极强大的可操作性，成为当 下热门的基因编辑工具. 近些年, CRISPR/Cas9 系统广 泛应用在 HIV-1 防治中: CRISPR/Cas9 基因编辑技术能 够通过靶向编辑 CCR 5 基因座位、直接清除 HIV-1 原病 毒或介导活化 HIV-1 等方式最终实现将患者体内 HIV-1 彻底根除 ${ }^{[101-102]}$

\section{3 休克和杀戮(Shock and Kill)}

利用 “Shock and Kill” 疗法治疗 HIV 潜伏库, 即利 用药物将被迫休眠的、受感染的细胞变得活跃，以便身 体自身的免疫系统或抗逆转录病毒药物杀死病毒, 最终 清除 HIV 病毒库 ${ }^{[103]}$. 现已经有许多尝试使用这种技术, HIV 潜伏库激活剂(Latency-reversing agent, LRAs)是研 
究主要方向, 然而到目前为止, 常用的 LRAs 可能无法 激活全部的 HIV 潜伏库, 因此必须研究出高度特异性的 LRAs 并考虑更多的新方法消除潜伏库 ${ }^{[104]}$.

除此之外, 还存在着干细胞移植治疗、广谱中和抗 体治疗、特异性 $\mathrm{T}$ 细胞治疗以及中药防治等治疗途径, 科学家们不断努力探索新的 HIV 治疗方法以求找到 HIV 完全治愈的可能.

\section{3 结论及展望}

过去十年来, 由于 ART 的出现, 新增的 HIV 感染者 的数量呈现缓慢下降的趋势. 一线和二线抗逆转录病毒 药物被广泛应用于治疗艾滋病, 现已展现出显著效果, 延缓了患病进程, 提升了患者的生活状态, 使艾滋病从 致死性疾病变成慢性感染性疾病, 但是药物的不良反 应、耐药性以及昂贵治疗费用也给病人和国家造成严重 的负担. 在不能够彻底根除 HIV 病毒潜伏库的现状下, 长效抗病毒药物的研发成为抗艾滋病药物研究重点. 为 了有效提高患者服药依从性并极大程度抑制病毒复制 以提高治疗效果, 合理联合用药、改善药物制剂的生物 以及物理活性、研究潜在的长效抗病毒药物制剂以及发 现全新靶点药物是未来抗艾滋病药物的研究方向.

\section{References}

[1] World Health Organization. HIV/AIDS [EB/OL] (2020-07-06). https://www.who.int/en/news-room/fact-sheets/detail/hiv-aids.

[2] Klatzmann, D.; Barre-Sinoussi, F.; Nugeyre, M. T.; Danquet, C.; Vilmer, E.; Griscelli, C.; Brun-Veziret, F.; Rouzioux, C.; Gluckman, J. C.; Chermann, J. C.; Montagnier, L. Science 1984, 225, 59.

[3] Esbjornsson, J.; Mansson, F.; Kvist, A.; da Silva, Z. J.; Andersson, S.; Fenyo, E. M.; Isberg, P. E.; Biague, A. J.; Lindman, J.; Palm, A. A.; Rowland-Jones, S. L.; Jansson, M.; Medstrand, P.; Norrgren, H.; Sweden and Guinea-Bissau Cohort Research Group. Lancet HIV 2019, 6, 25.

[4] Fanales-Belasio, E.; Raimondo, M.; Suligoi, B.; Butto, S. Ann. Ist. Super. Sanita 2010, 46, 5 .

[5] Richter, S. N.; Frasson, I.; Palu, G. Curr. Med. Chem. 2009, 16, 267.

[6] Shum, K. T.; Zhou, J.; Rossi, J. J. Pharmaceuticals 2013, 6, 1507.

[7] Gelderblom, H. R.; Ozel, M.; Pauli, G. Arch. Virol. 1989, 106, 1.

[8] Palmisano, L.; Vella, S. Ann. Ist. Super. Sanita 2011, 47, 44.

[9] Castro, S.D.; Camarasa, M. J. Eur. J. Med. Chem. 2018, 150, 206.

[10] Kleinpeter, A. B.; Freed, E. O. Viruses 2020, 12, 940.

[11] Kakuda, T. N. Clin. Ther. 2000, 22, 685.

[12] Ruane, P. J.; DeJesus, E.; Berger, D.; Markowitz, M.; Bredeek, U. F.; Callebaut, C.; Zhong, L.; Ramanathan, S.; Rhee, M. S.; Fordyce, M. W.; Yale, K. J. Acquired Immune Defic. Syndr. 2013, 63, 449.

[13] Ohrui, H. Chem. Rec. 2006, 6, 133.

[14] Ohrui, H.; Kohgo, S.; Hayakawa, H.; Kodama, E.; Matsuoka, M.; Nakata, T.; Mitsuya, H. Nucleosides, Nucleotides Nucleic Acids 2007, 26, 1543 .

[15] Kawamoto, A.; Kodama, E.; Sarafianos, S. G.; Sakagami, Y.; Kohgo, S.; Kitano, K.; Ashida, N.; Iwai, Y.; Hayakawa, H.; Nakata, H.; Mitsuya, H.; Arnold, E.; Matsuoka, M. Int. J. Biochem. Cell Biol. 2008, 40, 2410.

[16] Kirby, K. A.; Michailidis, E.; Fetterly, T. L.; Steinbach, M. A.; Singh, K.; Marchand, B.; Leslie, M. D.; Hagedorn, A. N.; Kodama, E. N.; Marquez, V. E.; Hughes, S. H.; Mitsuya, H.; Parniak, M. A.; Sarafianos, S. G. Antimicrob. Agents Chemother. 2013, 57, 6254.
[17] Muftuoglu, Y.; Sohl, C. D.; Mislak, A. C.; Mitsuya, H.; Sarafianos, S. G.; Anderson, K. S. Antiviral Res. 2014, 106, 1.

[18] Michailidis, E.; Marchand, B.; Kodama, E. N.; Singh, K.; Matsuoka, M.; Kirby, K. A.; Ryan, E. M.; Sawani, A. M.; Nagy, E.; Ashida, N.; Mitsuya, H.; Parniak, M. A.; Sarafianos, S. G. J. Biol. Chem. 2009, 284, 35681.

[19] Salie, Z. L.; Kirby, K. A.; Michailidis, E.; Marchand, B.; Singh, K.; Rohan, L. C.; Kodama, E. N.; Mitsuya, H.; Parniak, M. A.; Sarafianos, S. G. Proc. Natl. Acad. Sci. U. S. A. 2016, 113, 9274.

[20] McLaughlin, M.; Kong, J.; Belyk, K. M.; Chen, B.; Gibson, A. W.; Keen, S. P.; Lieberman, D. R.; Milczek, E. M.; Moore, J. C.; Murray, D.; Peng, F.; Qi, J.; Reamer, R. A.; Song, Z. J.; Tan, L.; Wang, L.; Williams, M. J. Org. Lett. 2017, 19, 926.

[21] Nawrat, C. C.; Whittaker, A. M.; Huffman, M. A.; McLaughlin, M.; Cohen, R. D.; Andreani, T.; Ding, B.; Li, H.; Weisel, M.; Tschaen, D. M. Org. Lett. 2020, 22, 2167.

[22] Michailidis, E.; Ryan, E. M.; Hachiya, A.; Kirby, K. A.; Marchand, B.; Leslie, M. D.; Huber, A. D.; Ong, Y. T.; Jackson, J. C.; Singh, K.; Kodama, E. N.; Mitsuya, H.; Parniak, M. A.; Sarafianos, S. G. Retrovirology 2013, 10, 65 .

[23] Stoddart, C. A.; Galkina, S. A.; Joshi, P.; Kosikova, G.; Moreno, M. E.; Rivera, J. M.; Sloan, B.; Reeve, A. B.; Sarafianos, S. G.; Murphey-Corb, M.; Parniak, M. A. Antimicrob. Agents Chemother. 2015, 59, 4190.

[24] Schurmann, D.; Rudd, D. J.; Zhang, S.; De Lepeleire, I.; Robberechts, M.; Friedman, E.; Keicher, C.; Huser, A.; Hofmann, J.; Grobler, J. A.; Stoch, S. A.; Iwamoto, M.; Matthews, R. P. Lancet HIV 2020, 7, 164.

[25] Markowitz, M.; Gettie, A.; St Bernard, L.; Andrews, C. D.; Mohri, H.; Horowitz, A.; Grasperge, B. F.; Blanchard, J. L.; Niu, T.; Sun, L.; Fillgrove, K.; Hazuda, D. J.; Grobler, J. A. J. Infect. Dis. 2020, $211,1398$.

[26] Markowitz, M.; Sarafianos, S. G. Curr. Opin. HIV AIDS 2018, 13, 294.

[27] Mackman, R. L.; Ray, A. S.; Hui, H. C.; Zhang, L.; Birkus, G.; Boojamra, C. G.; Desai, M. C.; Douglas, J. L.; Gao, Y.; Grant, D.; Laflamme, G.; Lin, K. Y.; Markevitch, D. Y.; Mishra, R.; McDermott, M.; Pakdaman, R.; Petrakovsky, O. V.; Vela, J. E.; Cihlar, T. Bioorg. Med. Chem. 2010, 18, 3606.

[28] Nie, B.; Jin, C.-F.; Zhong, W.-H.; Ren, Q.-Y.; Zhang, Y.-J.; Zhang, J. Chin. J. Org. Chem. 2017, 37, 2818 (in Chinese).

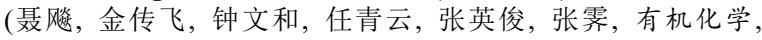
2017, 37, 2818.)

[29] Liu, Y.-C.; Cheng, J.-F.; Hong, R. Chin. J. Org. Chem. 2020, 40, 3237 (in Chinese).

(刘神辰, 程杰飞, 洪然, 有机化学, 2020, 40, 3237.)

[30] Cihlar, T.; Ray, A. S.; Boojamra, C. G.; Zhang, L.; Hui, H.; Laflamme, G.; Vela, J. E.; Grant, D.; Chen, J.; Myrick, F.; White, K. L.; Gao, Y.; Lin, K. Y.; Douglas, J. L.; Parkin, N. T.; Carey, A.; Pakdaman, R.; Mackman, R. L. Antimicrob. Agents Chemother. 2008, 52, 655 .

[31] Boojamra, C. G.; Mackman, R. L.; Markevitch, D. Y.; Prasad, V.; Ray, A. S.; Douglas, J.; Grant, D.; Kim, C. U.; Cihlar, T. Bioorg. Med. Chem. Lett. 2008, 18, 1120.

[32] Scarth, B. J.; White, K. L.; Chen, J. M.; Lansdon, E. B.; Swaminathan, S.; Miller, M. D.; Gotte, M. Antimicrob. Agents Chemother. 2011, 55, 2662.

[33] Lansdon, E. B.; Samuel, D.; Lagpacan, L.; Brendza, K. M.; White, K. L.; Hung, M.; Liu, X.; Boojamra, C. G.; Mackman, R. L.; Cihlar, T.; Ray, A. S.; McGrath, M. E.; Swaminathan, S. J. Mol. Biol. 2010, 397, 967.

[34] Zdanowicz, M. M. Am. J. Pharm. Educ. 2006, 70, 100.

[35] (a) Bastos, M. M.; Costa, C.; Bezerra, T. C.; da Silva, F. C.; Boechat, N. Eur. J. Med. Chem. 2016, 108, 455.

(b) Young, S. D.; Britcher, S. F.; Tran, L. O.; Payne, L. S.; Lumma, W. C.; Lyle, T. A.; Huff, J. R.; Anderson, P. S.; Olsen, D. B.; Carroll, S. S. Antimicrob. Agents Chemother. 1995, 39, 2602.

(c) Kawai, H.; Kitayama, T.; Tokunaga, E.; Shibata, N. Eur. J. Org. 
Chem. 2011, 30, 5959.

[36] Zheng, Y.; Zhang, L.; Meggers, E. Org. Process Res. Dev. 2018, 22, 103.

[37] Cote, B.; Burch, J. D.; Asante-Appiah, E.; Bayly, C.; Bedard, L.; Blouin, M.; Campeau, L. C.; Cauchon, E.; Chan, M.; Chefson, A.; Coulombe, N.; Cromlish, W.; Debnath, S.; Deschenes, D.; Dupont-Gaudet, K.; Falgueyret, J. P.; Forget, R.; Gagne, S.; Gauvreau, D.; Girardin, M.; Guiral, S.; Langlois, E.; Li, C. S.; Nguyen, N.; Papp, R.; Plamondon, S.; Roy, A.; Roy, S.; Seliniotakis, R.; St-Onge, M.; Ouellet, S.; Tawa, P.; Truchon, J. F.; Vacca, J.; Wrona, M.; Yan, Y.; Ducharme, Y. Bioorg. Med. Chem. Lett. 2014, 24, 917.

[38] Gauthier, D. R. Jr.; Sherry, B. D.; Cao, Y.; Journet, M.; Humphrey, G.; Itoh, T.; Mangion, I.; Tschaen, D. M. Org. Lett. 2015, 17, 1353.

[39] Lai, M. T.; Feng, M.; Falgueyret, J. P.; Tawa, P.; Witmer, M.; DiStefano, D.; Li, Y.; Burch, J.; Sachs, N.; Lu, M.; Cauchon, E.; Campeau, L. C.; Grobler, J.; Yan, Y.; Ducharme, Y.; Cote, B.; Asante-Appiah, E.; Hazuda, D. J.; Miller, M. D. Antimicrob. Agents Chemother. 2014, 58, 1652.

[40] Ren, J.; Bird, L. E.; Chamberlain, P. P.; Stewart-Jones, G. B.; Stuart, D. I.; Stammers, D. K. Proc. Natl. Acad. Sci. U. S. A. 2002, 99, 14410.

[41] Sanchez, R. I.; Fillgrove, K. L.; Yee, K. L.; Liang, Y.; Lu, B.; Tatavarti, A.; Liu, R.; Anderson, M. S.; Behm, M. O.; Fan, L.; Li, Y.; Butterton, J. R.; Iwamoto, M.; Khalilieh, S. G. Xenobiotia 2019, 49, 422.

[42] Rock, A. E.; Lerner, J.; Badowski, M. E. HIVIAIDS 2020, 12, 201.

[43] Orkin, C.; Squires, K. E.; Molina, J. M.; Sax, P. E.; Wong, W. W.; Sussmann, O.; Kaplan, R.; Lupinacci, L.; Rodgers, A.; Xu, X.; Lin, G.; Kumar, S.; Sklar, P.; Nguyen, B. Y.; Hanna, G. J.; Hwang, C.; Martin, E. A. Clin. Infect. Dis. 2019, 68, 535.

[44] Al-Salama, Z. T. Drugs 2017, 77, 1811.

[45] Namasivayam, V.; Vanangamudi, M.; Kramer, V. G.; Kurup, S.; Zhan, P.; Liu, X.; Kongsted, J.; Byrareddy, S. N. J. Med. Chem. 2018, 62, 4851.

[46] Rai, M. A.; Pannek, S.; Fichtenbaum, C. J. Expert Opin. Emerging Drugs 2018, 23, 149.

[47] Brik, A.; Wong, C. H. Org. Biomol. Chem. 2003, 1, 5.

[48] McKeage, K.; Perry, C. M.; Keam, S. J. Drugs 2009, 69, 477.

[49] Tashima, K.; Crofoot, G.; Tomaka, F. L.; Kakuda, T. N.; Brochot, A.; Van de Casteele, T.; Opsomer, M.; Garner, W.; Margot, N.; Custodio, J. M.; Fordyce, M. W.; Szwarcberg, J. AIDS Res. Ther. 2014, 11, 39.

[50] Kozisek, M.; Lepsik, M.; Grantz saskova, K.; Brynda, J.; Konvalinka, J.; Rezacova, P. FEBS J. 2014, $281,1834$.

[51] Huang, D.; Caflisch, A. J. Chem. Theory Comput. 2012, 8, 1786.

[52] Hohlfeld, K.; Wegner, J. K.; Kesteleyn, B.; Linclau, B.; Unge, J. J. Med. Chem. 2015, 58, 4029.

[53] Agniswamy, J.; Louis, J. M.; Shen, C. H.; Yashchuk, S.; Ghosh, A. K.; Weber, I. T. J. Med. Chem. 2015, 58, 5088.

[54] Ghosh, A. K.; Williams, J. N.; Ho, R. Y.; Simpson, H. M.; Hattori, S. I.; Hayashi, H.; Agniswamy, J.; Wang, Y. F.; Weber, I. T.; Mitsuya, H. J. Med. Chem. 2018, 61, 9722

[55] Ghosh, A. K.; Martyr, C. D.; Kassekert, L. A.; Nyalapatla, P. R.; Steffey, M.; Agniswamy, J.; Wang, Y. F.; Weber, I. T.; Amano, M.; Mitsuya, H. Org. Biomol. Chem. 2015, 13, 11607.

[56] Amano, M.; Miguel Salcedo-Gomez, P.; Yedidi, R. S.; Delino, N. S.; Nakata, H.; Venkateswara Rao, K.; Ghosh, A. K.; Mitsuya, H. Sci. Rep. 2017, 7, 12235.

[57] Strom, T. A.; Durdagi, S.; Ersoz, S. S.; Salmas, R. E.; Supuran, C. T.; Barron, A. R. J. Pept. Sci. 2015, 21, 862.

[58] Barzegar, A.; Jafari Mousavi, S.; Hamidi, H.; Sadeghi, M. Phys. E (Amsterdam, Neth.) 2017, 93, 324.

[59] Yasuno, T.; Ohe, T.; Kataoka, H.; Hashimoto, K.; Ishikawa, Y.; Furukawa, K.; Tateishi, Y.; Kobayashi, T.; Takahashi, K.; Nakamura, S.; Mashino, T. Bioorg. Med. Chem. Lett. 2021, 31, 127675.

[60] Liu, F.; Wang, Y.; Zhang, K.; Wang, Y.; Zhou, R.; Zeng, Y.; Han, Y.; Ng, T. B. Int. J. Biol. Macromol. 2017, 97, 339.
[61] Chen, M. H.; Chang, S. S.; Dong, B.; Yu, L. Y.; Wu, Y. X.; Wang R. Z.; Jiang, W.; Gao, Z. P.; Si, S. Y. RSC $A d v . \mathbf{2 0 1 8 , ~ 8 , ~} 5138$.

[62] Gao, K.; Butler, S. L.; Bushman, F. EMBO J. 2001, 20, 3565.

[63] Wang, H.; Kowalski, M. D.; Lakdawala, A. S.; Vogt, F. G.; Wu, L. Org. Lett. 2015, 17, 564.

[64] Margolis, D. A.; Boffito, M. Curr. Opin. HIV AIDS 2015, 10, 246.

[65] Trezza, C.; Ford, S. L.; Spreen, W.; Pan, R.; Piscitelli, S. Curr. Opin. HIV AIDS 2015, 10, 239.

[66] Spreen, W. R.; Margolis, D. A.; Pottage, J. C. Jr. Curr. Opin. HIV AIDS 2013, 8, 565.

[67] Murray, M.; Antela, A.; Mills, A.; Huang, J.; Jager, H.; Bernal, E.; Lombaard, J.; Katner, H.; Walmsley, S.; Khuong-Josses, M. A.; Hudson, K.; Dorey, D.; Griffith, S.; Spreen, W.; Vanveggel, S.; Shaefer, M.; Margolis, D.; Chounta, V. AIDS Behav. 2020, 24, 3533.

[68] Andrews, C. D.; Yueh, Y. L.; Spreen, W. R.; St Bernard, L.; BoenteCarrera, M.; Rodriguez, K.; Gettie, A.; Russell-Lodrigue, K.; Blanchard, J.; Ford, S.; Mohri, H.; Cheng-Mayer, C.; Hong, Z.; Ho, D. D.; Markowitz, M. Sci. Transl. Med. 2015, 7, 270 ra4.

[69] Mostashari Rad, T.; Saghaie, L.; Fassihi, A. Chem. Biodiversity 2018, 15, e1800159.

[70] Melikyan, G. B. J. Biol. Chem. 2017, 292, 16511.

[71] Markham, A. Drugs 2020, 80, 1615.

[72] Pancera, M.; Lai, Y. T.; Bylund, T.; Druz, A.; Narpala, S.; O'Dell, S.; Schon, A.; Bailer, R. T.; Chuang, G. Y.; Geng, H.; Louder, M. K.; Rawi, R.; Soumana, D. I.; Finzi, A.; Herschhorn, A.; Madani, N.; Sodroski, J.; Freire, E.; Langley, D. R.; Mascola, J. R.; McDermott, A. B.; Kwong, P. D. Nat. Chem. Biol. 2017, 13, 1115.

[73] Meanwell, N. A.; Krystal, M. R.; Nowicka-Sans, B.; Langley, D. R.; Conlon, D. A.; Eastgate, M. D.; Grasela, D. M.; Timmins, P.; Wang, T.; Kadow, J. F. J. Med. Chem. 2018, 61, 62.

[74] Nowicka-Sans, B.; Gong, Y. F.; McAuliffe, B.; Dicker, I.; Ho, H. T.; Zhou, N.; Eggers, B.; Lin, P. F.; Ray, N.; Wind-Rotolo, M.; Zhu, L.; Majumdar, A.; Stock, D.; Lataillade, M.; Hanna, G. J.; Matiskella, J. D.; Ueda, Y.; Wang, T.; Kadow, J. F.; Meanwell, N. A.; Krystal, M. Antimicrob. Agents Chemother. 2012, 56, 3498.

[75] Hiryak, K.; Koren, D. E. Ann. Pharmacother. 2021, 55, 792

[76] Blair, H. A. Drugs 2020, 80, 189.

[77] Nakane, S.; Iwamoto, A.; Matsuda, Z. J. Biol. Chem. 2015, 290, 15279 .

[78] Beccari, M. V.; Mogle, B. T.; Sidman, E. F.; Mastro, K. A.; Asiago-Reddy, E.; Kufel, W. D. Antimicrob. Agents Chemother. 2019, 63, e00110.

[79] Freeman, M. M.; Seaman, M. S.; Rits-Volloch, S.; Hong, X.; Kao, C. Y.; Ho, D. D.; Chen, B. Structure 2010, 18, 1632.

[80] Pace, C. S.; Fordyce, M. W.; Franco, D.; Kao, C. Y.; Seaman, M. S.; Ho, D. D. J. Acquired Immune Defic. Syndr. 2013, 62, 1

[81] Emu, B.; Fessel, J.; Schrader, S.; Kumar, P.; Richmond, G.; Win, S.; Weinheimer, S.; Marsolais, C.; Lewis, S. N. Engl. J. Med. 2018, 379, 645 .

[82] Bettiker, R. L.; Koren, D. E.; Jacobson, J. M. Curr. Opin. HIV AIDS 2018, 13, 354 .

[83] Thompson, D. A.; Cormier, E. G.; Dragic, T. J. Virol. 2002, 76, 3059 .

[84] Woollard, S. M.; Kanmogne, G. D. Drug Des. Dev. Ther. 2015, 9, 5447.

[85] Tan, Q.; Zhu, Y.; Li, J.; Chen, Z.; Han, G. W.; Kufareva, I.; Li, T.; Ma, L.; Fenalti, G.; Li, J.; Zhang, W.; Xie, X.; Yang, H.; Jiang, H.; Cherezov, V.; Liu, H.; Stevens, R. C.; Zhao, Q.; Wu, B. Science 2013, 341, 1387

[86] Zhu, Y.; Li, H.; Lin, K.; Wang, B.; Zhou, W. Synth. Commun. 2019, 49, 1721.

[87] Peng, P.; Chen, H.; Zhu, Y.; Wang, Z.; Li, J.; Luo, R. H.; Wang, J.; Chen, L.; Yang, L. M.; Jiang, H.; Xie, X.; Wu, B.; Zheng, Y. T.; Liu, H. J. Med. Chem. 2018, 61, 9621.

[88] Luo, W.; Srinivasulu, C.; Hao, X.; Liu, X.; Zhan, P. Expert Opin. Drug Discovery 2020, 15, 1115.

[89] Chan, D. C.; Fass, D.; Berger, J. M.; Kim, P. S. Cell 1997, 89, 263. 
[90] Ding, X.; Zhang, X.; Chong, H.; Zhu, Y.; Wei, H.; Wu, X.; He, J.; Wang, X.; He, Y. J. Virol. 2017, 91, e00831.

[91] Patel, I. H.; Zhang, X.; Nieforth, K.; Salgo, M.; Buss, N. Clin. Pharmacokinet. 2005, 44, 175.

[92] Chong, H.; Yao, X.; Zhang, C.; Cai, L.; Cui, S.; Wang, Y.; He, Y. PLoS One 2012, 7, e32599.

[93] Zhang, H.; Jin, R.; Yao, C.; Zhang, T.; Wang, M.; Xia, W.; Peng, H.; Wang, X.; Lu, R.; Wang, C.; Xie, D.; Wu, H. AIDS Res. Ther. 2016, 13,8 .

[94] Cambou, M. C.; Landovitz, R. J. Curr. HIV/AIDS Rep. 2020, 17, 118.

[95] Ganser, B. K.; Li, S.; Klishko, V. Y.; Finch, J. T.; Sundquist, W. I. Science 1999, 283, 80

[96] Link, J. O.; Rhee, M. S.; Tse, W. C.; Zheng, J.; Somoza, J. R.; Rowe, W.; Begley, R.; Chiu, A.; Mulato, A.; Hansen, D.; Singer, E.; Tsai, L. K.; Bam, R. A.; Chou, C. H.; Canales, E.; Brizgys, G.; Zhang, J. R.; Li, J.; Graupe, M.; Morganelli, P.; Liu, Q.; Wu, Q.; Halcomb, R. L.; Saito, R. D.; Schroeder, S. D.; Lazerwith, S. E.; Bondy, S.; Jin, D.; Hung, M.; Novikov, N.; Liu, X.; Villasenor, A. G.; Cannizzaro, C. E.; Hu, E. Y.; Anderson, R. L.; Appleby, T. C.; 㮡䠀, B.; Mwangi, J.; Liclican, A.; Niedziela-Majka, A.; Papalia, G. A.; Wong, M. H.; Leavitt, S. A.; Xu, Y.; Koditek, D.; Stepan, G. J.;
Yu, H.; Pagratis, N.; Clancy, S.; Ahmadyar, S.; Cai, T. Z.; Sellers, S.; Wolckenhauer, S. A.; Ling, J.; Callebaut, C.; Margot, N.; Ram, R. R.; Liu, Y. P.; Hyland, R.; Sinclair, G. I.; Ruane, P. J.; Crofoot, G. E.; McDonald, C. K.; Brainard, D. M.; Lad, L.; Swaminathan, S.; Sundquist, W. I.; Sakowicz, R.; Chester, A. E.; Lee, W. E.; Daar, E. S.; Yant, S. R.; Cihlar T. Nature 2020, 584, 614.

[97] Kleinpeter, A. B.; Freed, E. O. Viruses 2020, 12, 940.

[98] Johnson, M.; Jewell, R. C.; Peppercorn, A.; Gould, E.; Xu, J.; Lou, Y.; Davies, M.; Baldwin, S.; Tenorio, A. R.; Burke, M.; Jeffrey, J.; Johns, B. A. Pharmacol. Res. Perspect. 2018, 6, e00408.

[99] DeJesus, E.; Harward, S.; Jewell, R. C.; Johnson, M.; Dumont, E.; Wilches, V.; Halliday, F.; Talarico, C. L.; Jeffrey, J.; Gan, J.; Xu, J.; Felizarta, F.; Scribner, A.; Ramgopal, M.; Benson, P.; Johns, B. A. Clin. Infect. Dis. 2020, $71,1255$.

[100] Wen, Z.; Sun, C. Vaccines 2020, 8, 511.

[101] Hashmat, R.; Yousaf, M. Z.; Rahman, Z.; Anjum, K. M.; Yaqoob, A.; Imran, M. Crit. Rev. Eukaryotic Gene Expression 2020, 30, 77.

[102] Xiao, Q.; Guo, D.; Chen, S. Front. Cell. Infect. Microbiol. 2019, 9 , 69.

[103] Deeks, S. G. Nature 2012, 487, 439.

[104] Abner, E.; Jordan, A. Antiviral Res. 2019, 166, 19. 